CONFORMAL GEOMETRY AND DYNAMICS

An Electronic Journal of the American Mathematical Society

Volume 8, Pages 115-142 (June 8, 2004)

S $1088-4173(04) 00108-0$

\title{
BERS EMBEDDING OF THE TEICHMÜLLER SPACE OF A ONCE-PUNCTURED TORUS
}

\author{
YOHEI KOMORI AND TOSHIYUKI SUGAWA
}

\begin{abstract}
In this note, we present a method of computing monodromies of projective structures on a once-punctured torus. This leads to an algorithm numerically visualizing the shape of the Bers embedding of a one-dimensional Teichmüller space. As a by-product, the value of the accessory parameter of a four-times punctured sphere will be calculated in a numerical way as well as the generators of a Fuchsian group uniformizing it. Finally, we observe the relation between the Schwarzian differential equation and Heun's differential equation in this special case.
\end{abstract}

\section{INTRODUCTION}

The Teichmüller space was, as its name tells us, invented by O. Teichmüller around 1940. At this early stage, however, quasiconformal mappings had not been developed enough, and therefore, his work was difficult for most people to understand. In the late 1950 's, L. V. Ahlfors and L. Bers established a firm foundation to the theory of quasiconformal mappings, which led to a better understanding of Teichmüller space and the further development of it. Among their contributions, the idea of simultaneous uniformization due to Bers enabled us to embed the Teichmüller space of a Riemann surface as a bounded domain in the normed vector space of holomorphic quadratic differentials on it. In the latter half of the last century, many authors have revealed a number of remarkable properties of Teichmüller spaces. Most of them, however, are concerned with the internal geometry of the spaces. On the one hand, in connection with the deformation of Kleinian groups, the boundary of the Bers embedding (the so-called Bers boundary) of a Teichmüller space has been investigated. In spite of much effort in this direction, the shape of the Bers embedding is still mysterious at present because of its highly transcendental nature. At least, the supporting evidence that the Bers boundary is fairly irregular has been reported by, for instance, 22] and [38]. We also note that the Teichmüller space of finite dimension greater than one is not a symmetric domain. Actually, the analytic automorphism group of it is the homomorphic image of the mapping class group on the surface under the discontinuous action on the Teichmüller space

Received by the editors November 6, 2003 and, in revised form, March 16, 2004.

2000 Mathematics Subject Classification. Primary 30F60; Secondary 30F40, 34A20.

Key words and phrases. Teichmüller space, Bers embedding, monodromy, pleating ray, accessory parameter, bending coordinates, once-punctured torus.

The second author was partially supported by the Ministry of Education, Grant-in-Aid for Encouragement of Young Scientists, 9740056. A portion of the present research was carried out during the second author's visit to the University of Helsinki under the exchange program of scientists between the Academy of Finland and the JSPS.

(C)2004 American Mathematical Society 
(Royden [34]). As Bers stated in his survey [3], even one-dimensional Teichmüller spaces are exciting one's curiosity enough.

The first attempt to compute a boundary point of a one-dimensional Teichmüller space was made by R. M. Porter [32] and L. Keen, et al [13], 12, where they succeeded in drawing several real rays of trace functions ending at cusps in the case of the once-punctured square torus. The present authors are wondering why no one has developed their approach further despite the remarkable progress of computing technology in recent years. We note here that in the 1980's C. McMullen, D. Mumford and D. Wright obtained computer pictures of the Maskit embedding of the Teichmüller space of a once-punctured torus. Since the Maskit embedding had been expected to resemble the Bers embedding in shape, they made several conjectures on the Bers embedding (see, for instance, 25]). Moreover, the Bers boundary of a one-dimensional Teichmüller space was asserted to be a Jordan curve by C. McMullen around 1990. A proof for the assertion in the literature has been given by Y. Minsky as a corollary of his complete description of the space of discrete faithful representations of once-punctured torus groups [27.

Furthermore, using Minsky's Pivot Theorem, H. Miyachi [28] recently proved that each cusp of the one-dimensional Teichmüller space is really cusp-shaped; namely, to the endpoint of each rational pleating ray one can attach a subdomain like cardioid in the Bers embedding.

On the other hand, B. Maskit 24] (see also 21]) discovered projective structures such that the monodromies are quasi-Fuchsian groups but the developing maps are not univalent (for details of the terminology used here, see the next section). Those exotic projective structures have recently been studied intensively by many authors (see, e.g., [26] , [36], [11]). However, the configuration of components of the interior of exotic projective structures on a surface is still far from being clear.

In this article, we will give a practical method of computing the monodromy of a given projective structure by solving a linear ordinary differential equation of the second order which is associated with the Schwarzian differential equation. Employing the bending coordinates, which were developed by L. Keen and C. Series (see, e.g., [15]) and by C. McMullen [26], we explain how we can generate a picture of the Bers embedding with sufficient precision.

Our method can also be applied to obtain the whole picture of exotic components of the discreteness locus of projective structures and, if available, is an algorithm of determining the discreteness of a given Möbius group generated by two elements with parabolic commutator. This will be a main subject of our forthcoming paper [18] with M. Wada and Y. Yamashita.

This article is organized as follows. Section 2 is devoted to the basic definitions of Teichmüller spaces and related notions. In Section 3, we summarize known facts about commensurable Fuchsian groups with given Fuchsian group $\Gamma$ uniformizing a once-punctured torus. The explicit description of coverings between corresponding quotient surfaces will be helpful to transfer the computation on the once-punctured torus to that on the commensurable four-times punctured sphere in Section 4 In this way, we can avoid using elliptic functions in actual computations. This method can also be used to obtain exact values of the Poincaré density of the once-punctured square torus [37. Section 5 gives an enumeration of free homotopy classes of (non-peripheral, unoriented) simple closed curves on the topological oncepunctured torus by using the notion of Farey neighbours. In Section 6 we describe 
the natural "polar coordinates," called the bending coordinates, of the Teichmüller space of a once-punctured torus by following C. McMullen [26]. Our main theorem states that some sort of radial ray, called a rational pleating ray, can be described as a real locus of the entire function representing the trace of monodromy of a simple closed curve corresponding to the ray. Using results in the preceding sections, we can draw numerically all rational pleating rays in a recursive way. As a by-product, we can compute the value of the accessory parameter and a standard generator pair of a Fuchsian group uniformizing a given once-punctured torus as well. In the final section, we investigate the relation between the Schwarzian differential equation in the four-times punctured sphere and Heun's differential equation. We will observe that a simple change of indeterminants transforms the former one to the latter. Thus the monodromy can be computed also by solving Heun's differential equation instead of the Schwarzian differential equation.

Acknowledgments. The authors should like to express deep thanks to Yoshitsugu Takei and Shun Shimomura, who both pointed out the connection with Heun's differential equation. They also wish to thank Caroline Series and Hideki Miyachi for enjoyable conversations about Theorem 6.3 during the first author's stay at Warwick. Finally, the authors are grateful to the referee for his/her careful reading and thoughtful suggestions.

\section{BASIC DEFINITIONS}

Let $\Gamma$ be a Fuchsian group acting on the unit disk $\mathbb{D}$. In other words, $\Gamma$ is a discrete subgroup (possibly with torsion elements) of the analytic automorphism group $\operatorname{Aut}(\mathbb{D})$ of $\mathbb{D}$. Note that $\operatorname{Aut}(\mathbb{D})$ is Möbius conjugate to $\operatorname{Aut}(\mathbb{H}) \cong \operatorname{PSL}(2, \mathbb{R})$, where $\mathbb{H}$ denotes the upper half plane $\{z \in \mathbb{C} ; \operatorname{Im} z>0\}$. We denote by $Q(\mathbb{D}, \Gamma)$ the set of holomorphic quadratic differentials $\varphi(\zeta) d \zeta^{2}$ for $\Gamma$ on the unit disk, i.e., $\varphi$ are holomorphic in $\mathbb{D}$ and satisfy the functional equation $\varphi \circ \gamma \cdot\left(\gamma^{\prime}\right)^{2}=\varphi$ for each

$\gamma \in \Gamma$. (We often identify an element $\varphi(\zeta) d \zeta^{2}$ in $Q(\mathbb{D}, \Gamma)$ with the corresponding coefficient $\varphi(\zeta)$.)

A quadratic differential $\varphi(\zeta) d \zeta^{2} \in Q(\mathbb{D}, \Gamma)$ is called bounded if the norm

$$
\|\varphi\|_{\mathbb{D}}=\sup _{\zeta \in \mathbb{D}}\left(1-|\zeta|^{2}\right)^{2}|\varphi(\zeta)|
$$

is finite. We denote by $B_{2}(\mathbb{D}, \Gamma)$ the complex Banach space consisting of all bounded holomorphic quadratic differentials for $\Gamma$ on $\mathbb{D}$ with the norm defined above.

It is well known that $B_{2}(\mathbb{D}, \Gamma)$ is finite dimensional if and only if the Fuchsian group $\Gamma$ is cofinite; in other words, finitely generated and of the first kind. If $\Gamma$ is torsion-free and uniformizes a Riemann surface $X$ of finite analytic type $(g, n)$, where $g$ is the genus of $X$ and $n$ is the number of punctures of $X$ with $2 g-2+n>0$, then $\operatorname{dim} B_{2}(\mathbb{D}, \Gamma)=3 g-3+n$. In particular, $\operatorname{dim} B_{2}(\mathbb{D}, \Gamma)=1$ if and only if the signature $(g, n)$ is equal to $(1,1)$ or $(0,4)$. An element of $B_{2}(\mathbb{D}, \Gamma)$ is sometimes called a cusp form for a cofinite $\Gamma$. Each cusp form admits at most a simple pole at a puncture or a branch point of the quotient Riemann surface (or orbifold).

We now explain a well-known method enabling us to construct a locally univalent meromorphic function on $\mathbb{D}$ for which the Schwarzian derivative coincides with the given holomorphic quadratic differential. For a good account including the historical background, we refer the reader to [9, Chap. 10]. Let $\varphi \in Q(\mathbb{D}, \Gamma)$ be given. 
Then consider the following linear homogeneous ordinary differential equation of the second order:

$$
2 \eta^{\prime \prime}+\varphi \eta=0 \quad \text { on } \quad \mathbb{D} .
$$

Since $\mathbb{D}$ is simply connected, a unique solution $\eta$ exists on $\mathbb{D}$ for the given initial data $\eta(0)=a$ and $\eta^{\prime}(0)=b$. We denote by $\eta_{1}$ and $\eta_{2}$ the special solutions of (2.1) determined by $\eta_{1}(0)=0, \eta_{1}^{\prime}(0)=1$ and $\eta_{2}(0)=1, \eta_{2}^{\prime}(0)=0$. Then the Wronskian $\eta_{1}^{\prime} \eta_{2}-\eta_{1} \eta_{2}^{\prime}$ is identically 1 . We note that the ratio $f:=\eta_{1} / \eta_{2}$ has Taylor expansion in the form

$$
f(\zeta)=\zeta+a_{3} \zeta^{3}+\cdots
$$

near the origin. A simple calculation shows that the Schwarzian derivative $S_{f}=$ $\left(f^{\prime \prime} / f^{\prime}\right)^{\prime}-\left(f^{\prime \prime} / f^{\prime}\right)^{2} / 2$ is equal to the given differential $\varphi$. Hereafter, we denote by $f_{\varphi}$ the meromorphic function $f=\eta_{1} / \eta_{2}$ just described above and call it the (normalized) developing map of $\varphi$ when we regard $\varphi$ as a projective structure on the surface $\mathbb{D} / \Gamma$.

Here we briefly recall facts about the analytic automorphism group Aut $(\mathbb{D})$ of the unit disk $\mathbb{D}$. Let $\mathrm{SU}(1,1)$ be the subgroup of $\mathrm{SL}(2, \mathbb{C})$ consisting of all matrices $U$ in the form $\left(\begin{array}{ll}u & v \\ \bar{v} & \bar{u}\end{array}\right)$ with $|u|^{2}-|v|^{2}=1$. Then the canonical projection $\mathrm{SU}(1,1) \rightarrow \mathrm{PSU}(1,1)=\mathrm{SU}(1,1) /\{ \pm I\}$ gives the natural homomorphism $P: \mathrm{SU}(1,1) \rightarrow \operatorname{Aut}(\mathbb{D})$. In other words, $P(U)(z)=(u z+v) /(\bar{v} z+\bar{u})$. It is well known that $\mathrm{SU}(1,1)$ is conjugate with $\mathrm{SL}(2, \mathbb{R})$ in $\mathrm{SL}(2, \mathbb{C})$.

We define the pullback $U_{-1 / 2}^{*} \eta$ of $\eta$ under $U$ by

$$
U_{-1 / 2}^{*} \eta(z)=(\bar{v} z+\bar{u}) \eta(P(U)(z)) .
$$

Here we remark that the function $\bar{v} z+\bar{u}$ is a global branch of $\left(P(U)^{\prime}\right)^{-1 / 2}$.

A direct computation shows that $U_{-1 / 2}^{*} \eta$ satisfies differential equation (2.1) with $P(U)_{2}^{*} \varphi=\varphi \circ P(U) \cdot\left(P(U)^{\prime}\right)^{2}$ instead of $\varphi$. In particular, when $P(U) \in \Gamma$, we see that $U_{-1 / 2}^{*} \eta_{j}$ is again a solution of (2.1) for $j=1,2$, and hence, we can write

$$
\begin{aligned}
& U_{-1 / 2}^{*} \eta_{1}=a \eta_{1}+b \eta_{2}, \\
& U_{-1 / 2}^{*} \eta_{2}=c \eta_{1}+d \eta_{2},
\end{aligned}
$$

where $a, b, c$ and $d$ are constants, because $\eta_{1}$ and $\eta_{2}$ form a basis of the vector space of solutions of (2.1). (The relations in (2.2) can be regarded as "additive laws" for the solutions.) We note that we can deduce $a d-b c=1$ from the fact that the Wronskian of $U_{-1 / 2}^{*} \eta_{1}, U_{-1 / 2}^{*} \eta_{2}$ is identically 1 .

Let $\widetilde{\Gamma}$ be the inverse image $P^{-1}(\Gamma)$ of $\Gamma$ under the projection $P$. We now define the map $\tilde{\chi}_{\varphi}: \widetilde{\Gamma} \rightarrow \operatorname{SL}(2, \mathbb{C})$ by $\tilde{\chi}_{\varphi}(\tilde{\gamma})=\left(\begin{array}{ll}a & b \\ c & d\end{array}\right)$, where $a, b, c$ and $d$ are the above constants for $U=\tilde{\gamma} \in \widetilde{\Gamma}$. It is easy to see that $\tilde{\chi}_{\varphi}$ is actually a group homomorphism for each $\varphi$. By the holomorphic dependence of solutions on the coefficients, the correspondence $\varphi \mapsto \tilde{\chi}_{\varphi}(\tilde{\gamma})$ is a holomorphic map from $Q(\mathbb{D}, \Gamma)$ to $\operatorname{SL}(2, \mathbb{C})$, where the space $Q(\mathbb{D}, \Gamma)$ is endowed with the Fréchet space structure determined by the uniform convergence on compacta. Note that $\chi_{0}$ is nothing but the identity. Furthermore, for a $\tilde{\gamma} \in \widetilde{\Gamma}$, we set

$$
\operatorname{Tr}_{\tilde{\gamma}}(\varphi)=\operatorname{tr} \tilde{\chi}_{\varphi}(\tilde{\gamma})=a+d .
$$


The trace function $\operatorname{Tr}_{\tilde{\gamma}}$ is also holomorphic in $\varphi$ and plays an important role in the present investigation.

Taking the ratio of relations (2.2), we obtain

$$
f_{\varphi} \circ \gamma=\chi_{\varphi}(\gamma) \circ f_{\varphi}
$$

on $\mathbb{D}$, where $\gamma=P(\tilde{\gamma})$ and $\chi_{\varphi}(\gamma)$ is the Möbius transformation induced by $\tilde{\chi}_{\varphi}(\tilde{\gamma})$, i.e., $(a z+b) /(c z+d)$. The element $\chi_{\varphi}(\gamma)$ is well defined because $(a z+b) /(c z+d)$ is independent of the choice of $\tilde{\gamma} \in P^{-1}(\gamma) \subset \widetilde{\Gamma}$. Thus, the map $\chi_{\varphi}: \Gamma \rightarrow \operatorname{PSL}(2, \mathbb{C})$ is also a group homomorphism and will be called the monodromy homomorphism of $\Gamma$ associated with $\varphi \in Q(\mathbb{D}, \Gamma)$.

By virtue of the following theorem, we see that by considering only the monodromy homomorphism we would lose nothing in the investigation of deformations of $\Gamma$.

Theorem A. Let $\Gamma$ be a cofinite Fuchsian group. For $\varphi, \psi \in B_{2}(\mathbb{D}, \Gamma)$, the coincidence $\chi_{\varphi}=\chi_{\psi}$ on $\Gamma$ implies $\varphi=\psi$.

This result traces back to Poincaré for the case when $\Gamma$ uniformizes a compact Riemann surface. The generalization above is due to I. Kra 19.

The (Bers embedded) Teichmüller space of a Fuchsian group $\Gamma$ will be denoted by $T(\Gamma)$ and defined as the set of those holomorphic quadratic differentials $\varphi \in$ $Q(\mathbb{D}, \Gamma)$ for which the developing maps $f_{\varphi}$ are univalent and admit quasiconformal extensions to $\widehat{\mathbb{C}}$ compatible with the action of $\Gamma$, where a quasiconformal extension $F$ of $f_{\varphi}$ is said to be compatible with $\Gamma$ if for each $\gamma \in \Gamma$ there exists a Möbius transformation $\gamma^{\prime}$ such that $F \circ \gamma=\gamma^{\prime} \circ F$ on $\widehat{\mathbb{C}}$.

Remark 2.1. For simplicity, we have adopted a slightly different definition of the Teichmüller space from the usual one. Our space $T(\Gamma)$ here means, in the standard definition, the Teichmüller space of the Fuchsian group acting on the exterior of the unit disk, or, more intuitively, the Teichmüller space of the mirror image of the surface $\mathbb{D} / \Gamma$. Therefore, the reader should refer with special care to another paper. For example, our standard generator pair $\theta_{0}, \theta_{\infty}$ (see Section 3 below) should be replaced by $\theta_{0}{ }^{-1}, \theta_{\infty}$.

When $\Gamma$ is the trivial group 1, $T(1)$ is called the universal Teichmüller space. By definition, we see that $T\left(\Gamma_{1}\right) \subset T\left(\Gamma_{2}\right)$ if $\Gamma_{1} \supset \Gamma_{2}$. In particular, the universal Teichmüller space contains all Teichmüller spaces of Fuchsian groups.

The Kraus-Nehari theorem says that $\|\varphi\|_{\mathbb{D}} \leq 6$ if $f_{\varphi}$ is univalent. Hence $T(\Gamma)$ is a bounded subset of $B_{2}(\mathbb{D}, \Gamma)$. We also note that the Ahlfors-Weill theorem implies $\left\{\varphi \in B_{2}(\mathbb{D}, \Gamma) ;\|\varphi\|_{\mathbb{D}}<2\right\} \subset T(\Gamma)$. It is also well known that $T(\Gamma)$ is a connected open subset of $B_{2}(\mathbb{D}, \Gamma)$. For details, see [10. By the existence of a conformally natural extension operator from quasisymmetric homeomorphisms of $\mathrm{S}^{1}$ to quasiconformal ones of $\mathbb{D}$ (see Tukia [39] or Douady-Earle [4]), we can see that

$$
T(\Gamma)=T(1) \cap B_{2}(\mathbb{D}, \Gamma)
$$

and that $T(\Gamma)$ is contractible.

More generally, we consider the set $K(\Gamma)$ of those quadratic differentials $\varphi \in$ $B_{2}(\mathbb{D}, \Gamma)$ for which the monodromy images $\chi_{\varphi}(\Gamma)$ are discrete in $\operatorname{PSL}(2, \mathbb{C})$. The following result is due to H. Shiga [35].

Theorem B. The Bers embedded Teichmüller space $T(\Gamma)$ coincides with the connected component of Int $K(\Gamma)$ which contains the origin. 
Moreover, each element of $\operatorname{Int} K(\Gamma)$ is known to correspond to an isomorphism onto a quasi-Fuchsian group (Shiga and Tanigawa [36]). Thus, the trace function $\operatorname{Tr}_{\tilde{\gamma}}$ for a hyperbolic element $\tilde{\gamma} \in \widetilde{\Gamma}$ satisfies

$$
\operatorname{Tr}_{\tilde{\gamma}}(\operatorname{Int} K(\Gamma)) \subset \mathbb{C} \backslash[-2,2] .
$$

By virtue of Theorem B, we would see the shape of the Bers embedding if we could mark the grids contained in $K(\Gamma)$ for sufficiently fine mesh in the space $B_{2}(\mathbb{D}, \Gamma)$. This approach is out of our scope here, however, it will be a basis of our forthcoming paper $[18$ in the case when $\mathbb{D} / \Gamma$ is a once-punctured torus. It also has the advantage that one can draw a picture (up to translation) even if one does not know about the value of the accessory parameter (see Section 5). To do that, we need an algorithm determining the discreteness of a given Möbius group. Such an algorithm, however, is available only for the special case when the group is generated by two elements with parabolic commutator at present.

Our method here will rely on the internal geometry of the Teichmüller space, and hence, it might be applicable to even higher dimensional cases.

\section{Commensurability Relations}

All one-dimensional Teichmüller spaces are mutually conformally equivalent because they are all simply connected. In particular, the Teichmüller space of a once-punctured torus is biholomorphic to that of a four-times punctured sphere. There is, however, an intrinsic relation between them as is described below.

Let $\Gamma$ be a Fuchsian group uniformizing a once-punctured torus $X$. It is a basic fact that $\Gamma$ has free generators $\theta_{0}$ and $\theta_{\infty}$ with the properties: (i) both are hyperbolic; (ii) the commutator $\left[\theta_{0}, \theta_{\infty}\right]$ is parabolic; and (iii) the (signed) intersection number $\theta_{0} \cdot \theta_{\infty}$ equals 1 . We call such $\left(\theta_{0}, \theta_{\infty}\right)$ a standard generator pair of $\Gamma$.

If a standard generator pair is given, then there exists a unique complex number $\tau$ with $\operatorname{Im} \tau>0$ satisfying the following property: The quotient surface $(\mathbb{C} \backslash\{m+n \tau ; m, n \in \mathbb{Z}\}) / L_{\tau}=\left(\mathbb{C} \backslash L_{\tau}\right) / L_{\tau}$, where $L_{\tau}$ is the lattice group generated by 1 and $\tau$ over $\mathbb{Z}$, is conformally equivalent to $X$ in such a way that the curves $t+(1+\tau) / 2$ and $t \tau+(1+\tau) / 2$ in $\mathbb{C}$ parametrized by $t \in[0,1]$ correspond to $\theta_{0}$ and $\theta_{\infty}$, respectively.

We call $\tau$ the Teichmüller parameter of the (marked) Fuchsian group $\Gamma$ with standard generator $\left(\theta_{0}, \theta_{\infty}\right)$. This parameter $\tau$ is known to give a global analytic coordinate of the Teichmüller space of $\Gamma$ onto the upper half plane $\mathbb{H}$ (see [10]).

Now consider the four-times punctured torus $Z$ given as the quotient $(\mathbb{C} \backslash\{(m+n \tau) / 2 ; m, n \in \mathbb{Z}\}) / L_{\tau}=\left(\mathbb{C} \backslash \frac{1}{2} L_{\tau}\right) / L_{\tau}$. Then the mapping $z \mapsto 2 z$ induces a four-sheeted (unbranched) covering $Z \rightarrow X$. Note that the covering group of $Z \rightarrow X$ is isomorphic to $\mathbb{Z}_{2} \oplus \mathbb{Z}_{2}$.

On the other hand, the conformal involution $z \mapsto-z$ induces a 2-sheeted (unbranched) covering $Z \rightarrow Y$, where $Y$ is the four-times punctured sphere described as $\widehat{\mathbb{C}} \backslash\left\{e_{0}, e_{1}, e_{2}, e_{3}\right\}$, where $e_{0}=\wp(0)=\infty, e_{1}=\wp(1 / 2), e_{2}=\wp(\tau / 2)$, $e_{3}=\wp((1+\tau) / 2)$, in terms of the Weierstrass $\wp$-function with period lattice $L_{\tau}$. We note that $e_{1}+e_{2}+e_{3}=0$.

Since the covering $Z \rightarrow X$ is Galois, we obtain a natural homomorphism from $\Gamma$ to $\operatorname{Aut}(Z)$. We denote by $\Gamma_{0}$ the kernel of this homomorphism. Then $\Gamma_{0}$ is a Fuchsian group uniformizing $Z$. By the above remark, we note that $\Gamma / \Gamma_{0} \cong \mathbb{Z}_{2} \oplus \mathbb{Z}_{2}$. 
The composition of the universal covering $\mathbb{D} \rightarrow \mathbb{D} / \Gamma_{0} \cong Z$ and the above projection $Z \rightarrow Y$ is a universal covering of the four-times punctured sphere $Y$, whose covering group will be denoted by $\Gamma^{\prime}$. Then $\Gamma^{\prime}$ is an extension of $\Gamma_{0}$ such that $\Gamma^{\prime} / \Gamma_{0} \cong \mathbb{Z}_{2}$.

Similarly, by the involution $z \mapsto-z$ of $\mathbb{C} \backslash\{m+n \tau ; m, n \in \mathbb{Z}\}$, we obtain a 2sheeted covering $X \rightarrow W$, where $W$ is a Riemann orbifold of signature $(0 ; 2,2,2, \infty)$. In fact, $W$ is realized as the complex plane with ramification of order 2 at the three points $e_{1}, e_{2}$ and $e_{3}$ via the covering map $[z] \mapsto \wp(z)$. This orbifold appears also as the quotient space of $Y$ by the action of the group $G\left(e_{0}, e_{1}, e_{2}, e_{3}\right)$ described below.

Let $a_{0}, a_{1}, a_{2}$ and $a_{3}$ be distinct points in the Riemann sphere $\widehat{\mathbb{C}}$ and set $\Omega=$ $\widehat{\mathbb{C}} \backslash\left\{a_{0}, a_{1}, a_{2}, a_{3}\right\}$. Unless these points are in a special position, we cannot permute these in an arbitrary way by a Möbius transformation. The domain $\Omega$, however, always has a special kind of symmetry.

For example, there exists a unique Möbius transformation $A_{1}$ swapping $a_{0}$ and $a_{1}$ and swapping $a_{2}$ and $a_{3}$. In fact, we take the Möbius transformation $A$ sending $a_{0}, a_{1}, a_{2}$ to $a_{1}, a_{0}, a_{3}$, respectively. Since $A$ swaps $a_{0}$ and $a_{1}$, the transformation $A$ must be an involution, i.e., $A^{2}=\mathrm{id}$. Therefore we conclude $A\left(a_{3}\right)=A^{-1}\left(a_{3}\right)=a_{2}$.

Similarly, we can take Möbius transformations $A_{2}$ and $A_{3}$ such that

$$
\begin{aligned}
& A_{2}\left(a_{0}, a_{1}, a_{2}, a_{3}\right)=\left(a_{2}, a_{3}, a_{0}, a_{1}\right), \\
& A_{3}\left(a_{0}, a_{1}, a_{2}, a_{3}\right)=\left(a_{3}, a_{2}, a_{1}, a_{0}\right) .
\end{aligned}
$$

We now have the group, denoted by $G\left(a_{0}, a_{1}, a_{2}, a_{3}\right)$, formed by the elements id, $A_{1}, A_{2}$ and $A_{3}$, which acts on $\Omega$ as analytic automorphisms and is isomorphic to $\mathbb{Z}_{2} \oplus \mathbb{Z}_{2}$. The quotient space $\Omega / G\left(a_{0}, a_{1}, a_{2}, a_{3}\right)$ is a Riemann orbifold of signature $(0 ; 2,2,2, \infty)$. We remark that the fixed points of $A_{1}$ can be expressed by

$$
\frac{a_{0} a_{1}-a_{2} a_{3} \pm \sqrt{\left(a_{0}-a_{2}\right)\left(a_{1}-a_{2}\right)\left(a_{0}-a_{3}\right)\left(a_{1}-a_{3}\right)}}{a_{0}+a_{1}-a_{2}-a_{3}} .
$$

We now return to our case. The covering map $R: Y \rightarrow Y / G\left(e_{0}, e_{1}, e_{2}, e_{3}\right)=W$ can be expressed explicitly by

$$
\begin{aligned}
R(z) & =\frac{z^{4}-2\left(e_{1} e_{2}+e_{2} e_{3}+e_{3} e_{1}\right) z^{2}+8 e_{1} e_{2} e_{3} z+\left(e_{1} e_{2}+e_{2} e_{3}+e_{3} e_{1}\right)^{2}}{4\left(z-e_{1}\right)\left(z-e_{2}\right)\left(z-e_{3}\right)} \\
& =\frac{z^{4}+g_{2} z^{2} / 2+2 g_{3} z+\left(g_{2} / 4\right)^{2}}{4 z^{3}-g_{2} z-g_{3}},
\end{aligned}
$$

where $g_{2}$ and $g_{3}$ are well-known constants determined by the lattice $L_{\tau}$. This rational function comes from the additive law in the theory of elliptic functions, i.e., $\wp(2 z)=R(\wp(z))$, and was used to construct an example of complex dynamics such that the Julia set equals the whole sphere $\widehat{\mathbb{C}}$ by Lattès in 1918 (see $\S 4.3$ of [2]).

Let $\Gamma_{1}$ be the covering group of the universal cover $\mathbb{D} \rightarrow X \rightarrow W$ of $W$. We note that $\Gamma_{0} \triangleleft \Gamma \triangleleft \Gamma_{1}, \Gamma_{0} \triangleleft \Gamma^{\prime} \triangleleft \Gamma_{1}, \Gamma_{0}=\Gamma \cap \Gamma^{\prime}$, and that $\Gamma_{1}=\left\langle\Gamma, \Gamma^{\prime}\right\rangle$.

In particular, $B_{2}\left(\mathbb{D}, \Gamma_{1}\right) \subset B_{2}(\mathbb{D}, \Gamma)$ and $B_{2}\left(\mathbb{D}, \Gamma_{1}\right) \subset B_{2}\left(\mathbb{D}, \Gamma^{\prime}\right)$, and these are all one-dimensional vector spaces; hence, all are equal. Now relation (2.4) leads to the following result.

Lemma 3.1. The Bers embedded one-dimensional Teichmüller spaces of the commensurable Fuchsian groups $\Gamma, \Gamma^{\prime}$ and $\Gamma_{1}$ all coincide:

$$
T(\Gamma)=T\left(\Gamma_{1}\right)=T\left(\Gamma^{\prime}\right) .
$$


Therefore, to consider the Bers embedding of the Teichmüller space of a oncepunctured torus is equivalent to also considering the corresponding four-times punctured sphere. The latter sometimes has the advantage of making things in the calculation simple, as we shall see in the next section.

\section{THE MONODROMY ON A FOUR-TIMES PUNCTURED SPHERE}

Let $\Gamma$ be the Fuchsian group uniformizing a once-punctured torus and $\Gamma^{\prime}$ be the Fuchsian group commensurable with $\Gamma$ uniformizing the corresponding fourtimes punctured sphere. Although the space $B_{2}(\mathbb{D}, \Gamma)=B_{2}\left(\mathbb{D}, \Gamma^{\prime}\right)$ is complex one-dimensional, it seems quite hard to calculate numerically the value of functions in this space. On the other hand, an element of $B_{2}(\mathbb{D}, \Gamma)$ can be regarded as a holomorphic quadratic differential on the quotient space $\mathbb{D} / \Gamma$, which is more tractable than its lift to $\mathbb{D}$. Indeed, R. M. Porter 32] and L. Keen [12 employed this idea and succeeded in computing the real rays of trace functions for several words of special type. In the authors' opinion, the appearance of elliptic functions in their methods has made the computation somewhat difficult.

So, the authors would like to propose an idea using quadratic differentials on the four-times punctured sphere $Y=\mathbb{D} / \Gamma^{\prime}$ instead of those on the once-punctured torus $X=\mathbb{D} / \Gamma$. Our formulation will need only rational functions, so the involved computation will be much simpler than the other methods. In this section, we will provide a method of computing the monodromy homomorphism $\chi_{\varphi}: \Gamma \rightarrow \operatorname{PSL}(2, \mathbb{C})$ up to conjugation by $\operatorname{PSL}(2, \mathbb{C})$, where the conjugation does not depend on $\varphi$. In fact, our construction gives global holomorphic mappings $\mu_{0}, \mu_{\infty}: B_{2}(\mathbb{D}, \Gamma) \rightarrow \operatorname{SL}(2, \mathbb{C})$ such that the homomorphism $\tilde{\chi}_{\varphi}^{\prime}: \widetilde{\Gamma} \rightarrow \operatorname{SL}(2, \mathbb{C})$ determined by $\tilde{\chi}_{\varphi}^{\prime}\left(\tilde{\theta}_{0}\right)=\mu_{0}(\varphi)$ and $\tilde{\chi}_{\varphi}^{\prime}\left(\tilde{\theta}_{\infty}\right)=\mu_{\infty}(\varphi)$ induces the same homomorphism $\Gamma \rightarrow \operatorname{PSL}(2, \mathbb{C})$ as $\chi_{\varphi}$ up to $\operatorname{PSL}(2, \mathbb{C})$-conjugacy (Theorem 4.3). Here $\tilde{\theta}_{r}$ is a representative of $\theta_{r}$ in $\operatorname{SU}(1,1)$ for $r=0, \infty$. We remark that the argument developed below can also be applied to the case of $n$-times punctured spheres, $n>4$.

Recall the relation $\mathbb{D} / \Gamma^{\prime} \cong \mathbb{C} \backslash\left\{e_{1}, e_{2}, e_{3}\right\}$. For simplicity, by the map $z \mapsto$ $\left(z-e_{2}\right) /\left(e_{1}-e_{2}\right)$, we transform the domain to the other one $\mathbb{C} \backslash\{0,1, \lambda\}$, denoted still by the same letter $Y$ as before, where $\lambda=\left(e_{3}-e_{2}\right) /\left(e_{1}-e_{2}\right)=\lambda(\tau)$ is known to be a holomorphic universal covering of the domain $\mathbb{C} \backslash\{0,1\}$ and usually called an elliptic modular function (cf. 1, Chapter 7]).

We denote by $p$ the universal covering $\mathbb{D} \rightarrow Y \cong \mathbb{D} / \Gamma^{\prime}$ constructed above and set $z_{0}=p(0)$. Then we can associate the isomorphism, denoted by $\delta$, from $\Gamma^{\prime}$ onto $\pi_{1}\left(Y, z_{0}\right)$ with $p$ by assigning the homotopy class of the image $p([0, \gamma(0)])$ to a given $\gamma \in \Gamma^{\prime}$

Let $\rho_{Y}(z)|d z|$ denote the complete hyperbolic metric on $Y$ of the constant negative curvature -4 , i.e., $1 /\left(1-|\zeta|^{2}\right)=\rho_{Y}(p(\zeta))\left|p^{\prime}(\zeta)\right|$. A holomorphic quadratic differential $\psi(z) d z^{2}$ on $Y$ is said to be bounded if the norm

$$
\|\psi\|_{Y}:=\sup _{z \in Y}|\psi(z)| \rho_{Y}(z)^{-2}
$$

is finite. We denote by $B_{2}(Y)$ the Banach space of bounded holomorphic quadratic differentials on $Y$. We remark that a holomorphic quadratic differential $\psi$ is bounded on $Y$ if and only if $\psi$ has at most simple poles at the punctures of $Y$. 
By definition, the spaces $B_{2}\left(\mathbb{D}, \Gamma^{\prime}\right)$ and $B_{2}(Y)$ are isometrically isomorphic via the pullback $p_{2}^{*}: B_{2}(Y) \rightarrow B_{2}\left(\mathbb{D}, \Gamma^{\prime}\right)$ defined by $p_{2}^{*} \psi=\psi \circ p \cdot\left(p^{\prime}\right)^{2}$. In particular, $B_{2}(Y)$ is one-dimensional, too.

On the other hand, the rational function

$$
\psi_{0}(z)=\frac{1}{z(z-1)(z-\lambda)}
$$

belongs to $B_{2}(Y)$, thus, $\psi_{0}$ forms a basis of the vector space $B_{2}(Y)$.

Now we consider the developing map $f_{\varphi}: \mathbb{D} \rightarrow \widehat{\mathbb{C}}$, where $\varphi$ is the pullback of a given $\psi \in B_{2}(Y)$ under the projection $p$. Then the (local) branch $g$ of the function $f_{\varphi} \circ p^{-1}$ near the basepoint $z_{0}=p(0)$ with $g\left(z_{0}\right)=0$ satisfies the relation

$$
p_{2}^{*} \psi\left(=\varphi=S_{f_{\varphi}}\right)=p_{2}^{*}\left(S_{g}\right)+S_{p} .
$$

The function $g$ has the expansion

$$
g(z)=\frac{1}{p^{\prime}(0)}\left(z-z_{0}\right)-\frac{p^{\prime \prime}(0)}{2 p^{\prime}(0)^{3}}\left(z-z_{0}\right)^{2}+\cdots
$$

around the point $z_{0}$. We should remember the fact that the first two coefficients do not depend on the particular choice of $\varphi=p_{2}^{*} \psi$. Here the relation $S_{p}=-p_{2}^{*}\left(S_{p^{-1}}\right)$ holds and $S_{p^{-1}}$ depends only on the domain $Y$; in fact, this is independent of the choice of the branch and the covering map $p$ because of the invariance property of the Schwarzian derivative: $S_{A \circ f}=S_{f}$ for any Möbius transformation $A$. This holomorphic quadratic differential $S_{p^{-1}}(z) d z^{2}$ will be written by $\nu_{Y}(z) d z^{2}$ in this article and called the uniformizing connection of $Y$ (see [20]).

By the behaviour of the universal covering near the puncture, the quadratic differential $\nu_{Y}(z) d z^{2}$ is known to have a pole of the second order at every puncture of $Y$ with residue 1/2. By this observation, we have the following.

Lemma 4.1 ([8, Theorem 3.1], 20, (2.3.1)]). The uniformizing connection $\nu_{Y}(z) d z^{2}$ of $Y$ has the form

$$
\nu_{Y}(z)=\frac{1}{2 z^{2}(z-1)^{2}}+\frac{1}{2(z-\lambda)^{2}}+\frac{c(\lambda)}{z(z-1)(z-\lambda)},
$$

where $c(\lambda)$ is a constant determined by $\lambda$.

The constant $c(\lambda)$ was called the accessory parameter by Poincaré and is known to be difficult to compute in general. I. Kra [20] showed that $c(\lambda)$ is real analytic but not complex analytic in $\lambda$.

If a Möbius transformation $A$ leaves $Y$ invariant, we have $\nu_{Y}=A_{2}^{*} \nu_{Y}$, in particular, $\nu_{Y}$ is invariant under the action of the group $G(0,1, \infty, \lambda)$. By this invariance, when $Y$ has a good symmetry, the value $c(\lambda)$ can be computed explicitly. For example, we easily see that $c(\lambda)$ is real for a real $\lambda$ and that $c(\lambda)$ is purely imaginary if $\operatorname{Re} \lambda=1 / 2$. In particular, we see that $c(1 / 2)=0$. The following examples can be found in $[8]$.

Example 4.1. $c(-1)=1$. Note that $\lambda(-1+i)=-1$.

Example 4.2. $c((1+\sqrt{3} i) / 2)=-i / \sqrt{3}$. Note that $\lambda((1+\sqrt{3} i) / 2)=(1+\sqrt{3} i) / 2$.

For the moment, we proceed ahead as if we know the value of the accessory parameter $c(\lambda)$. We will return to this problem in the final part of Section [5. 
Since $S_{p}=-p_{2}^{*}\left(\nu_{Y}\right)$, we can see from (4.2) that $S_{g}$ is a globally defined quadratic differential in $Y$ :

$$
S_{g}=\psi+\nu_{Y}
$$

Now we write $\psi$ in the form $\psi=t \psi_{0}$, where $t$ is a complex number and $\psi_{0}$ is given by (4.1), and consider the linear ordinary differential equation on $Y$ of the following form:

$$
2 y^{\prime \prime}+\left\{\frac{1}{2 z^{2}(z-1)^{2}}+\frac{1}{2(z-\lambda)^{2}}+\frac{t+c(\lambda)}{z(z-1)(z-\lambda)}\right\} y=0 .
$$

We remark that I. Laine and T. Sorvali [23] investigated the general differential equation $y^{\prime \prime}+C y=0$ with coefficient $C$ meromorphic in a simply connected domain and obtained a nice condition under which the (local) monodromy of the equation becomes trivial (see Corollary 4.2 therein).

Introducing the auxiliary unknown function $x$, we can transform this equation to the system of linear ordinary differential equations of the first order:

$$
\mathbf{y}^{\prime}=\mathbf{y} A, \quad A=\left(\begin{array}{cc}
0 & 1 \\
-\frac{1}{2}\left(\psi+\nu_{Y}\right) & 0
\end{array}\right)
$$

where $\mathbf{y}=\left(\begin{array}{ll}x & y\end{array}\right)$.

For a given point $z_{1} \in Y$, let $\mathbf{y}_{1}=\left(\begin{array}{ll}x_{1} & y_{1}\end{array}\right)$ and $\mathbf{y}_{2}=\left(\begin{array}{ll}x_{2} & y_{2}\end{array}\right)$ be the (local) solutions of (4.6) near the point $z_{1}$ satisfying $\mathbf{y}_{1}\left(z_{1}\right)=\left(\begin{array}{ll}1 & 0\end{array}\right)$ and $\mathbf{y}_{2}\left(z_{1}\right)=\left(\begin{array}{ll}0 & 1\end{array}\right)$. Then the matrix

$$
F_{z_{1}}=\left(\begin{array}{l}
\mathbf{y}_{1} \\
\mathbf{y}_{2}
\end{array}\right)=\left(\begin{array}{ll}
y_{1}^{\prime} & y_{1} \\
y_{2}^{\prime} & y_{2}
\end{array}\right)
$$

will be called the fundamental matrix of differential equation (4.6) at $z_{1}$. Note that $\operatorname{det} F_{z_{1}} \equiv 1$. In particular, the matrix $F_{z_{1}}$ is a holomorphic map from a neighbourhood of $z_{1}$ in $Y$ to the complex Lie group SL $(2, \mathbb{C})$ with $F_{z_{1}}\left(z_{1}\right)=I$, where $I$ denotes the identity matrix. The matrix $F_{z_{1}}$ itself can be regarded as an $\mathrm{SL}(2, \mathbb{C})$-valued (local) solution of the differential equation (4.6). An arbitrary (local) solution $y$ of (4.5) near the point $z_{1}$ with the initial conditions $y^{\prime}\left(z_{1}\right)=a$ and $y\left(z_{1}\right)=b$ can be expressed by

$$
\left(\begin{array}{ll}
y^{\prime} & y
\end{array}\right)=\left(\begin{array}{ll}
a & b
\end{array}\right) F_{z_{1}}
$$

and vice versa.

The holomorphic map $F_{z_{1}}$ can be analytically continued along any path $\alpha$ from $z_{1}$ to $z_{2}$ in $Y$. The resulting germ at $z_{2}$ depends only on the homotopy class $[\alpha]$ of $\alpha$ in $Y$, and hence, will be denoted by $H_{[\alpha]}$. Since the germ $H_{[\alpha]}$ is an $\operatorname{SL}(2, \mathbb{C})$-valued local solution of (4.6) near the point $z_{2}$, there exists a unique constant matrix $B \in \mathrm{SL}(2, \mathbb{C})$ such that $H_{[\alpha]}=B F_{z_{2}}$. The matrix $B$ is called the transition matrix along $[\alpha]$ and will be denoted by $L[\alpha]$ or, more precisely, $L_{t}[\alpha]$. In what follows, we will denote by $Y\left[z_{1}, z_{2}\right]$ the set of homotopy classes of paths connecting $z_{1}$ and $z_{2}$ in $Y$.

By definition, we can see the following fundamental properties of the transition matrix:

(i) $H_{[\alpha]}=L[\alpha] F_{z_{2}}$ for $[\alpha] \in Y\left[z_{1}, z_{2}\right]$,

(ii) $L[\alpha]=H_{[\alpha]}\left(z_{2}\right)$ for $[\alpha] \in Y\left[z_{1}, z_{2}\right]$, and

(iii) $L[\alpha] L[\beta]=L[\alpha \cdot \beta]$ for $[\alpha] \in Y\left[z_{1}, z_{2}\right]$ and $[\beta] \in Y\left[z_{2}, z_{3}\right]$. 
Property (ii) means that the transition matrix $L[\alpha]$ can be computed by (numerically) solving differential equation (4.6) along the path $\alpha$. (In practical computations, a polygonal line should be taken as $\alpha$.)

We set $l_{t}[\alpha]=L_{t}[\alpha]$ for $[\alpha] \in \pi_{1}\left(Y, z_{0}\right)=Y\left[z_{0}, z_{0}\right]$. Then, by property (iii), the map $l_{t}: \pi_{1}\left(Y, z_{0}\right) \rightarrow \mathrm{SL}(2, \mathbb{C})$ is a group homomorphism and will be called the monodromy homomorphism associated with $\psi=t \psi_{0}$.

Now we consider the ratio $y_{1} / y_{2}$, which will be denoted by $h_{z_{1}}$, of $y_{1}$ and $y_{2}$ appearing in the fundamental matrix $F_{z_{1}}$ at $z_{1}$. In other words, $h_{z_{1}}=\Pi \circ F_{z_{1}}$, where $\Pi: \operatorname{SL}(2, \mathbb{C}) \rightarrow \mathbb{C} \mathrm{P}^{1}=\widehat{\mathbb{C}}$ is defined by $\Pi:\left(\begin{array}{ll}a & b \\ c & d\end{array}\right) \mapsto b / d$. Then $h_{z_{1}}$ satisfies the Schwarzian differential equation (4.4) and has expansion in the form $h(z)=\left(z-z_{1}\right)+b_{3}\left(z-z_{1}\right)^{3}+\cdots$ near the point $z_{1}$. In particular, when we take $z_{0}$ as the reference point, comparing the above expansion with (4.3), we obtain

$$
g=k \circ h, \quad \text { where } \quad k(z)=\frac{p^{\prime}(0)^{2} z}{p^{\prime}(0)-p^{\prime \prime}(0) z / 2} \quad \text { and } \quad h=h_{z_{0}} .
$$

Note that the Möbius transformation $k$ depends only on the universal covering $p$ of $Y$ and that the function $h$ can be analytically continued to the function $\bar{l}_{t}[\alpha] \circ h$ along a curve $\alpha$, where $\bar{l}_{t}[\alpha]$ is the Möbius transformation induced by $l_{t}[\alpha]$.

Let us look at the relation between $\chi_{t \varphi_{0}}$ and $l_{t}$ for $t \in \mathbb{C}$, where $\varphi_{0}=p_{2}^{*}\left(\psi_{0}\right) \in$ $B_{2}\left(\mathbb{D}, \Gamma^{\prime}\right)$. First note that the monodromy homomorphism $\chi_{t \varphi_{0}}$ is defined over the Fuchsian group $\Gamma_{1}=\left\langle\Gamma, \Gamma^{\prime}\right\rangle$ because $B_{2}\left(\mathbb{D}, \Gamma^{\prime}\right)=B_{2}\left(\mathbb{D}, \Gamma_{1}\right)$. We also recall that $\delta: \Gamma^{\prime} \rightarrow \pi_{1}\left(Y, z_{0}\right)$ is the isomorphism mapping $\gamma$ to $p([0, \gamma(0)])$. Now we are ready to state the following theorem.

Theorem 4.2. The monodromies $\chi_{t \varphi_{0}}$ and $\bar{l}_{t}$ are essentially the same. More precisely,

$$
\chi_{t \varphi_{0}}(\gamma)=k \circ \bar{l}_{t}(\delta(\gamma)) \circ k^{-1}
$$

for each $t \in \mathbb{C}$ and $\gamma \in \Gamma^{\prime}$, where $\varphi_{0}=p_{2}^{*} \psi_{0}$ and $k$ is the Möbius transformation given in (4.7).

Proof. Set $g=k \circ h$, where $h=h_{z_{0}}$. Then, as was explained, $f=g \circ p$ near the origin. By the analytic continuation along the image curve $\alpha=\delta(\gamma)$ of the oriented hyperbolic geodesic segment joining 0 and $\gamma(0)$ in the unit disk $\mathbb{D}$, the function $h$ changes to $\bar{l}_{t}(\alpha) \circ h$. Hence, analytically continuing both sides of $f=k \circ h \circ p$ along this segment, we see $f=A \circ k \circ h \circ p=A \circ g \circ p$ near the point $\gamma(0)$, here we write $A=k \circ \bar{l}_{t}(\delta(\gamma)) \circ k^{-1}$ for short. In particular, $f \circ \gamma=A \circ g \circ p \circ \gamma=A \circ g \circ p=A \circ f$ near the origin. This implies $\chi_{\varphi}(\gamma)=A$.

Remark 4.1. The monodromy homomorphism $\chi_{\varphi}: \Gamma \rightarrow \operatorname{PSL}(2, \mathbb{C})$ has no natural homomorphic lifts $\Gamma \rightarrow \mathrm{SL}(2, \mathbb{C})$ unless we specify a homomorphic section $s: \Gamma \rightarrow$ $\mathrm{SU}(1,1) \subset \mathrm{SL}(2, \mathbb{C})$ of $\Gamma$. In contrast, $l_{t}: \pi_{1}\left(Y, z_{0}\right) \rightarrow \mathrm{SL}(2, \mathbb{C})$ is intrinsically homomorphic. Particularly, the composed map $l_{0} \circ \delta: \Gamma^{\prime} \rightarrow \mathrm{SU}(1,1)$ gives a homomorphic lift of the Fuchsian group $\Gamma^{\prime}$. As we can see from the standard local theory of Fuchsian differential equations around regular singular points, $\operatorname{tr}\left(l_{t}[\alpha]\right)=$ -2 holds for every simple loop $[\alpha]$ going around a puncture of $Y$. By this constraint, the lift of $\Gamma^{\prime}$ is uniquely determined (note that $\Gamma^{\prime}$ is a free group generated by three elements corresponding to punctures). Moreover, Y. Okumura [30] observes under this constraint that $\operatorname{tr}\left(l_{0}[\alpha]\right) \leq-2$ holds for each dividing simple loop $\alpha$ in $Y$ (even in more general context). This implies that the homomorphism $l_{0} \circ \delta$ has no 
homomorphic extensions to $\Gamma_{1}=\left\langle\Gamma, \Gamma^{\prime}\right\rangle$. In fact, if such an extension, say $\chi: \Gamma_{1} \rightarrow$ $\operatorname{SL}(2, \mathbb{R})$, exists, then it must satisfy $\operatorname{tr}\left(\chi\left(\gamma^{2}\right)\right)=\operatorname{tr}\left(\chi(\gamma)^{2}\right)=\operatorname{tr}(\chi(\gamma))^{2}-2 \geq 2$ for each element $\gamma \in \Gamma$. If we choose $\gamma$ to correspond to a non-peripheral simple closed curve in $X$, then $\gamma^{2} \in \Gamma^{\prime}$ corresponds to a dividing loop $\alpha$ in $Y$, and thus $\operatorname{tr}\left(\chi\left(\gamma^{2}\right)\right)=\operatorname{tr}\left(l_{0}[\alpha]\right)<-2$, which is a contradiction.

As is well known, for determination of a homomorphism $\chi: \Gamma \rightarrow \operatorname{PSL}(2, \mathbb{C})$, it is enough to know the values of $\operatorname{tr}^{2}(\chi(\gamma))$ for $\gamma=\theta_{0}, \theta_{\infty}$ and $\theta_{1}=\theta_{0} \theta_{\infty}$, where $\left(\theta_{0}, \theta_{1}\right)$ is a standard generator pair of $\Gamma$. Using Theorem 4.2, we can compute $\operatorname{tr}^{2}\left(\chi_{\varphi}\left(\theta_{r}\right)\right)$ for $r=0,1, \infty$. In fact, since $\delta\left(\theta_{r}^{2}\right)$ is a dividing loop in $Y$, we see $\operatorname{tr}^{2}\left(\chi_{\varphi}\left(\theta_{r}\right)\right)= \pm \operatorname{tr}\left(\chi_{\varphi}\left(\theta_{r}^{2}\right)\right)+2=-\operatorname{tr}\left(l_{t}\left(\delta\left(\theta_{r}^{2}\right)\right)\right)+2$ by the above remark. However, sometimes it is more convenient to have the representation $\chi_{\varphi}$ itself rather than just the traces. In order to directly compute $\chi_{\varphi}(\gamma)$ for each $\gamma \in \Gamma$ (up to Möbius conjugation) we use the symmetry of the domain $Y$ which was explained in the previous section.

Given a once-punctured torus $X=\mathbb{D} / \Gamma$ represented by the Teichmüller parameter $\tau$, it suffices to compute the monodromy images of the standard generator pair $\left(\theta_{0}, \theta_{\infty}\right)$ of $\Gamma$. The fact that $\varphi=p_{2}^{*} \psi \in B_{2}\left(\mathbb{D}, \Gamma_{1}\right)$ for each $\psi \in B_{2}(Y)$ implies that $\psi(z) d z^{2}$ is invariant under the action of $\Gamma_{1} / \Gamma^{\prime} \cong G(0,1, \infty, \lambda)<\operatorname{Aut}(Y)$, where $\lambda=\lambda(\tau)$. Since $\nu_{Y}(z) d z^{2}$ is also invariant, $A_{-1 / 2}^{*} y$ becomes a local solution of (4.5) for each local solution $y$ of (4.5) and for $A \in G(0,1, \infty, \lambda)$ by the same reasoning given in Section 2, where $A_{-1 / 2}^{*} y$ is defined by $y \circ A \cdot\left(A^{\prime}\right)^{-1 / 2}$ and $\left(A^{\prime}\right)^{-1 / 2}(z)=r z+s$ for a representative $\left(\begin{array}{ll}p & q \\ r & s\end{array}\right) \in \mathrm{SL}(2, \mathbb{C})$ of $A$. We set for $F_{A\left(z_{1}\right)}=\left(\begin{array}{ll}y_{1}^{\prime} & y_{1} \\ y_{2}^{\prime} & y_{2}\end{array}\right)$,

$$
A^{*}\left(F_{A\left(z_{1}\right)}\right):=\left(\begin{array}{ll}
\left(A_{-1 / 2}^{*} y_{1}\right)^{\prime} & A_{-1 / 2}^{*} y_{1} \\
\left(A_{-1 / 2}^{*} y_{2}\right)^{\prime} & A_{-1 / 2}^{*} y_{2}
\end{array}\right)=M_{z_{1}}(A) F_{z_{1}},
$$

where the matrix $M_{z_{1}}(A)$ is defined by

$$
M_{z_{1}}(A)=\left(\begin{array}{cc}
1 /\left(r z_{1}+s\right) & 0 \\
r & r z_{1}+s
\end{array}\right) .
$$

We consider the simple closed curves $\hat{\beta}_{0}$ and $\hat{\beta}_{\infty}$ in $Z$ induced by the curves $s+(1+\tau) / 4$ and $s \tau+(1+\tau) / 4$ parametrized by $s \in[0,1]$ in $\mathbb{C} \backslash \frac{1}{2} L_{\tau}$ and we take the reference point $z_{0}$ in $Y$ so that the intersection point of $\hat{\beta}_{0}$ and $\hat{\beta}_{\infty}$ projects to $z_{0}$ under the projection $Z \rightarrow Y$. Then the image curves $\beta_{0}$ and $\beta_{\infty}$ in $Y$ represent the homotopy classes $\delta\left(\theta_{0}^{2}\right)$ and $\delta\left(\theta_{\infty}^{2}\right)$, respectively.

Note that $\beta_{0}$ separates 0 and $\lambda$ from 1 and $\infty$. Hence we see that $A_{0}\left(z_{0}\right)=$ $p\left(\theta_{0}(0)\right)$, where $A_{0}(z)=(z-\lambda) /(z-1) \in G(0,1, \infty, \lambda)$ is determined by $A_{0}(0, \lambda, 1, \infty)$ $=(\lambda, 0, \infty, 1)$. Similarly, noting that $\beta_{\infty}$ separates 0 and $\infty$ from 1 and $\lambda$, we have $A_{\infty}\left(z_{0}\right)=p\left(\theta_{\infty}(0)\right)$, where $A_{\infty}(z)=\lambda / z \in G(0,1, \infty, \lambda)$ is determined by $A_{\infty}(0, \infty, 1, \lambda)=(\infty, 0, \lambda, 1)$. For $r=0, \infty$, let $\alpha_{r}$ be an oriented curve in $Y$ joining $z_{0}$ and $A_{r}\left(z_{0}\right)$ which is homotopic to $p_{*}\left(\left[0, \theta_{r}(0)\right]\right)$; in other words, homotopic to the curve coming from the one $s / 2+(1+\tau) / 4$ for $r=0$ or $s \tau / 2+(1+\tau) / 4$ for $r=\infty$ in $Z$ parametrized by $s \in[0,1]$.

Now we are in a position to state one of our main results.

Theorem 4.3. For suitable representatives $\tilde{\theta}_{0}$ of $\theta_{0}$ and $\tilde{\theta}_{\infty}$ of $\theta_{\infty}$ in $\mathrm{SU}(1,1)$,

$$
\tilde{\chi}_{t \varphi_{0}}\left(\tilde{\theta}_{0}\right)=L_{t}\left[\alpha_{0}\right] M_{z_{0}}\left(A_{0}\right), \quad \text { and } \quad \tilde{\chi}_{t \varphi_{0}}\left(\tilde{\theta}_{\infty}\right)=L_{t}\left[\alpha_{\infty}\right] M_{z_{0}}\left(A_{\infty}\right)
$$


hold for all $t \in \mathbb{C}$ up to $\mathrm{SL}(2, \mathbb{C})$-conjugacy independent of $t$, where $\varphi_{0}=p_{2}^{*}\left(\psi_{0}\right)$ and $\psi_{0}$ is given by (4.1).

Remark 4.2. If the point $z_{0}$ and the paths $\alpha_{0}$ and $\alpha_{\infty}$ are replaced by other $z_{1} \in$ $Y, \alpha_{0}^{\prime} \in Y\left[z_{1}, A_{0}\left(z_{1}\right)\right]$ and $\alpha_{\infty}^{\prime} \in Y\left[z_{1}, A_{\infty}\left(z_{1}\right)\right]$, the above result remains true as far as $\alpha_{0}^{\prime} \cdot\left(A_{0}\right)_{*}\left(\alpha_{0}^{\prime}\right)$ and $\alpha_{\infty}^{\prime} \cdot\left(A_{\infty}\right)_{*}\left(\alpha_{\infty}^{\prime}\right)$ are freely homotopic to $\beta_{0}$ and $\beta_{\infty}$ in $Y$, respectively.

Proof. We set $\varphi=t \varphi_{0}$ for a fixed $t \in \mathbb{C}$ and let $f=f_{\varphi}: \mathbb{D} \rightarrow \widehat{\mathbb{C}}$ be the normalized developing map for $\varphi$. Then $f=k \circ h_{z_{0}} \circ p$ near the origin of $\mathbb{D}$, where $k$ is the Möbius transformation given by (4.7). Let $r$ represent 0 or $\infty$. Analytically continuing both sides along the segment $\left[0, \theta_{r}(0)\right]$ in $\mathbb{D}$, we obtain

$$
f=k \circ L_{t}\left[\alpha_{r}\right]^{-} \circ h_{z_{0}^{\prime}} \circ p
$$

near the point $\theta_{r}(0)$, where we set $z_{0}^{\prime}=A_{r}\left(z_{0}\right)$ and denote by $M^{-}$the Möbius transformation induced by a matrix $M \in \mathrm{GL}(2, \mathbb{C})$. Noting $h_{z_{0}^{\prime}} \circ A_{r}=M_{z_{0}}\left(A_{r}\right)^{-} \circ$ $h_{z_{0}}$ by (4.9) and $p \circ \theta_{r}=A_{r} \circ p$, we have

$$
\begin{aligned}
f \circ \theta_{r} & =k \circ L_{t}\left[\alpha_{r}\right]^{-} \circ h_{z_{0}^{\prime}} \circ p \circ \theta_{r}=k \circ L_{t}\left[\alpha_{r}\right]^{-} \circ h_{z_{0}^{\prime}} \circ A_{r} \circ p \\
& =k \circ L_{t}\left[\alpha_{r}\right]^{-} \circ M_{z_{0}}\left(A_{r}\right)^{-} \circ h_{z_{0}} \circ p=k \circ L_{t}\left[\alpha_{r}\right]^{-} \circ M_{z_{0}}\left(A_{r}\right)^{-} \circ k^{-1} \circ f
\end{aligned}
$$

near the origin. This implies $\chi_{\varphi}\left(\theta_{r}\right)=k \circ L_{t}\left[\alpha_{r}\right]^{-} \circ M_{z_{0}}\left(A_{r}\right)^{-} \circ k^{-1}$. Hence, $\tilde{\chi}_{\varphi}\left(\tilde{\theta}_{r}\right)= \pm \tilde{k} L_{t}\left[\alpha_{r}\right] M_{z_{0}}\left(A_{r}\right) \tilde{k}^{-1}$, where $\tilde{k}$ is a matrix representing $k$. Since the quantities $\tilde{\chi}_{t \varphi_{0}}\left(\tilde{\theta}_{r}\right)$ and $L_{t}\left[\alpha_{r}\right] M_{z_{0}}\left(A_{r}\right)$ are globally holomorphic in $t$, we easily see that the above sign is constant. (Note also that the conjugacy map $k$ does not depend on $t$.) Therefore, we obtain the required equalities by choosing a suitable sign of $\tilde{\theta}_{r}$ for each $r$.

Remark 4.3. The following relation might be useful: For a point $z_{1}$ in $Y$ and $A \in G(0,1, \infty, \lambda)$, we set $z_{2}=A\left(z_{1}\right)$. Then we have $L[\alpha] M_{z_{1}}=M_{z_{2}} L\left[A_{*}(\alpha)\right]$ for any path $\alpha$ connecting $z_{1}$ and $z_{2}$ in $Y$.

We note also that we can use the orbifold $W$ instead of $Y$ in order to compute $\chi_{\varphi}\left(\theta_{r}\right)$ directly. The present method, however, has the merit that we can "save" the polygonal path used in solving the differential equation numerically. If we try to solve the differential equation along a closed polygonal path, then we would need more segments.

\section{Enumeration of SImPle Closed CuRVES}

In the preceding section, we have presented a method of computing the monodromy for a given projective structure and $\gamma \in \Gamma$. We explain here how to compute the values of the trace function corresponding to an arbitrary simple closed geodesic in a recursive and algebraic way using the particular values $\operatorname{Tr}_{\tilde{\theta}_{0}}, \operatorname{Tr}_{\tilde{\theta}_{\infty}}$ and $\operatorname{Tr}_{\tilde{\theta}_{1}}$, where $\tilde{\theta}_{r}$ is a fixed representative of $\theta_{r}$ in $\mathrm{SU}(1,1)$ for each $r$. The materials here are, more or less, known although rigorous proofs for those are sometimes difficult to locate. The reader is referred to, e.g., [15], [27] and [40] for further discussions. A good introduction to the Teichmüller space of a torus can also be found in [10].

Since we need only the topological structure of a once-punctured torus for a while, we treat first the topological once-punctured torus described as $\Sigma=\left(\mathbb{R}^{2} \backslash \mathbb{Z}^{2}\right) / \mathbb{Z}^{2}$. We denote by $\langle z\rangle$ the projection of a point $z \in \mathbb{R}^{2} \backslash \mathbb{Z}^{2}$ to $\Sigma$. Let $[\alpha]$ and $[\beta]$ be the homotopy classes in $\Sigma$ with basepoint $x=\langle(1 / 2,1 / 2)\rangle$ which are represented by 
the curves $\alpha(t)=x+\langle(t, 0)\rangle$ and $\beta(t)=x+\langle(0, t)\rangle, 0 \leq t \leq 1$, respectively. Let $A$ and $B$ be the homology classes corresponding to $[\alpha]$ and $[\beta]$. It is well known that the fundamental group $\pi_{1}(\Sigma, x)$ is a free group generated by $[\alpha]$ and $[\beta]$ and that the homology group $H_{1}(\Sigma)$ is a free abelian group generated by $A$ and $B$. Taking $A$ and $B$ as a basis, we may regard $H_{1}(\Sigma)$ as the lattice group $\mathbb{Z} \oplus \mathbb{Z}$.

The fundamental group and the homology group coincide for the unpunctured torus $\bar{\Sigma}=\mathbb{R}^{2} / \mathbb{Z}^{2}$ and both are isomorphic to $\mathbb{Z}^{2}=\mathbb{Z} \oplus \mathbb{Z}$. In particular, the homomorphism $\pi_{1}(\Sigma, x) \rightarrow \pi_{1}(\bar{\Sigma}, x)$ induced by the natural inclusion $\Sigma \rightarrow \bar{\Sigma}$ is canonically isomorphic to the abelianization $\pi_{1}(\Sigma, x) \rightarrow H_{1}(\Sigma)=H_{1}(\bar{\Sigma})$.

A simple closed curve $\gamma$ in $\Sigma$ is said to be non-peripheral if $\gamma$ can be continuously shrunken to neither a point nor a puncture in $\Sigma$. A non-peripheral simple closed curve $\gamma$ corresponds to a homology class of the form $p A+q B$, where $p$ and $q$ are relatively prime integers; that is, $\{m p+n q ; m, n \in \mathbb{Z}\}=\mathbb{Z}$. We can easily understand this as follows; cutting $\Sigma$ along $\gamma$, we have a once-punctured annulus, which implies that there is another non-peripheral simple closed curve $\gamma^{\prime}$ such that homology classes of $\gamma$ and $\gamma^{\prime}$ generate $H_{1}(\Sigma)$, hence the homology class of $\gamma$ must be of the above form.

The ratio $p / q$ will be called the slope of $\gamma$. Note that the slope does not depend on the orientation of the curve.

Remark 5.1. The reader should be careful with the term "slope" when referring to another paper devoted to the subject similar to ours because some authors prefer to call $-q / p$ the slope of $\gamma$. The reason is explained by the fact that the pinching deformation of a once-punctured torus along the curve $\gamma$ corresponds to letting the Teichmüller parameter $\tau \in \mathbb{H}$ tend to the point $-q / p \in \partial \mathbb{H}$ (see [17] for details). In this article, however, we adopt $r=p / q$ as the slope so that $r$ represents the inclination of the vector $(p, q)$.

As might be seen from the linear action of $\operatorname{SL}(2, \mathbb{Z})$ on $\mathbb{R}^{2}$ leaving $\mathbb{Z}^{2}$ invariant, the mapping class group of $\Sigma$ is isomorphic to $\mathrm{SL}(2, \mathbb{Z})$. This is a classical result. However, this could be seen more easily with a stronger assertion about the relation between simple closed curves and their slope.

Let us note here that the mapping class group $\mathcal{M}(\Sigma)$ of $\Sigma$ is defined as Homeo $^{+}(\Sigma) / \mathrm{Homeo}_{0}(\Sigma)$, where $\mathrm{Homeo}^{+}(\Sigma)$ is the group of orientation-preserving self-homeomorphisms of $\Sigma$ and $\mathrm{Homeo}_{0}(\Sigma)$ is the subgroup consisting of all homeomorphisms which are homotopic to the identity in $\Sigma$. We also remark that the mapping class group of $\Sigma$ is isomorphic to the quotient of the automorphism group of $\pi_{1}(\Sigma, x)$ over the inner automorphism group of $\pi_{1}(\Sigma, x)$.

Proposition 5.1. The mapping class group of $\Sigma$ is canonically isomorphic to $\mathrm{SL}(2, \mathbb{Z})$. For each rational $r \in \widehat{\mathbb{Q}}$ there exists a non-peripheral simple closed curve in $\Sigma$ with slope $r$. If two non-peripheral simple closed curves in $\Sigma$ have the same slope, they are freely homotopic in $\Sigma$ up to orientation.

The latter assertion can be found in 15 .

Proof. Let $S$ be the homeomorphism of $\Sigma$ defined by $S(\langle(s, t)\rangle)=\langle(s, t)\rangle$ for $0 \leq$ $t \leq 1 / 2$ and by $S(\langle(s, t)\rangle)=\langle(s+2 t-1, t)\rangle$ for $1 / 2 \leq t \leq 1$, which realizes a Dehn twist around the curve $\alpha$. Also let $T$ be the rotation $\langle(s, t)\rangle \mapsto\langle(t,-s)\rangle$ by $\pi / 2$. Then the induced homomorphisms $S_{*}$ and $T_{*}$ on $\pi_{1}(\Sigma, x)$ satisfy $S_{*}(\alpha, \beta)=(\alpha, \alpha \beta)$ 
and $T_{*}(\alpha, \beta)=\left(\beta, \alpha^{-1}\right)$, and hence, they act on $H_{1}(\Sigma)$ as matrices $\left(\begin{array}{ll}1 & 1 \\ 0 & 1\end{array}\right)$ and $\left(\begin{array}{cc}0 & -1 \\ 1 & 0\end{array}\right)$, respectively, under the above identification with $\mathbb{Z} \oplus \mathbb{Z}$. It is well known that the above two matrices generate $\mathrm{SL}(2, \mathbb{Z})$, and thus, the natural homomorphism $\mathcal{M}(\Sigma) \rightarrow \operatorname{Aut}\left(H_{1}(\Sigma)\right) \cong \mathrm{SL}(2, \mathbb{Z})$ is surjective.

A rational $r \in \widehat{\mathbb{Q}}$ can be written in the form $r=p / q$, where $p$ and $q$ are relatively prime integers. Then there exist integers $p^{\prime}$ and $q^{\prime}$ such that $p q^{\prime}-p^{\prime} q=1$. We now take an element $f_{*}$ in $\mathcal{M}(\Sigma)$ which acts on $H_{1}(\Sigma)$ as the matrix $\left(\begin{array}{ll}p & p^{\prime} \\ q & q^{\prime}\end{array}\right)$. Then $f_{*}(\alpha)$ has slope $r$ as desired.

We next show the uniqueness part. By means of the action of the mapping class group, it is enough to show that a simple closed curve $\alpha^{*}$ in $\pi_{1}(\Sigma, x)$ with slope 0 is necessarily homotopic to $\alpha$ in $\Sigma$. Since $\Sigma \backslash \alpha^{*}$ is a punctured annulus, we can connect both boundary components by a simple arc with endpoints at $x$. That arc can be regarded as a simple closed curve, say $\beta^{*}$, in $\Sigma$ so that $\alpha^{*}$ and $\beta^{*}$ form standard generators of $\pi_{1}(\Sigma, x)$. Furthermore, by repeated application of the Dehn twist along $\alpha^{*}$, we can choose $\beta^{*}$ so that it is homotopic to $\beta$ in $\bar{\Sigma}$.

If we take an orientation-preserving self-homeomorphism $f$ of $\Sigma$ fixing the point $x$ such that $f_{*}(\alpha)=\alpha^{*}$ and $f_{*}(\beta)=\beta^{*}$, then $f_{*}$ acts on $\pi_{1}(\bar{\Sigma}, x)$ trivially. Therefore, $f$ is homotopic to the identity in the unpunctured torus $\bar{\Sigma}$. Let $f_{t}$ be an isotopy in $\bar{\Sigma}$ connecting the identity and $f$ with $f_{0}=$ id and with $f_{1}=f$. Since $\bar{\Sigma}=\mathbb{R}^{2} / \mathbb{Z}^{2}$ is an abelian group, $\hat{f}_{t}=f_{t}-f_{t}(0)$ is an isotopy in $\bar{\Sigma}$ fixing the origin $0=\langle(0,0)\rangle$. Hence $\left.\hat{f}_{t}\right|_{\Sigma}$ is an isotopy in $\Sigma$ connecting the identity and $f$, which implies that $\alpha^{*}$ is freely homotopic to $\alpha$.

Finally, we show the first assertion in the proposition. It suffices to prove the injectivity of the natural homomorphism $\mathcal{M}(\Sigma) \rightarrow \operatorname{Aut}\left(H_{1}(\Sigma)\right)=\mathrm{SL}(2, \mathbb{Z})$. Let $f_{*}$ be in the kernel of the above homomorphism. We may assume that $f$ fixes $x$. Then $f_{*}(\alpha)$ and $f_{*}(\beta)$ have slope 0 and $\infty$, respectively. The last argument yields also that $f$ is homotopic to the identity in $\Sigma$. Hence $f_{*}$ is trivial on $\pi_{1}(\Sigma, x)$.

We can extract from the proposition the following corollary, which will be utilized effectively below.

Corollary 5.2. Let $\left(\gamma_{1}, \gamma_{2}\right)$ be a standard generator pair of the fundamental group $\pi_{1}(\Sigma, x)$ of the topological once-punctured torus $\Sigma$. Then $\left(\gamma_{1}, \gamma_{1} \gamma_{2}\right),\left(\gamma_{1} \gamma_{2}, \gamma_{2}\right)$ and $\left(\gamma_{2}, \gamma_{1}^{-1}\right)$ are all standard generator pairs of $\pi_{1}(\Sigma, x)$.

We next recall fundamental facts about Farey triangles (cf. [27]). The reader also finds a good account for Farey sequences in [7] as well as an interesting historical remark.

For three points $a, b, c$ in $\widehat{\mathbb{R}}$, we denote by $\Delta(a, b, c)$ the hyperbolic triangle formed by three hyperbolic geodesics in the upper half plane $\mathbb{H}$ connecting two of the three points $a, b$ and $c$. Let $\Delta=\Delta(0,1, \infty)$. Then $\mathbb{H}$ is tessellated by $\Delta$ and its conjugates by the modular group $\operatorname{PSL}(2, \mathbb{Z})$. Note that the stabilizer of $\Delta$ in $\operatorname{PSL}(2, \mathbb{Z})$ consists of three elements and permutes the vertices of $\Delta$ cyclically. Each triangle which is conjugate with $\Delta$ by the action of $\operatorname{PSL}(2, \mathbb{Z})$ is called a Farey triangle. The initial Farey triangle $\Delta$ and its reflection $\Delta^{\prime}=\Delta(0,-1, \infty)$ in the imaginary axis form a fundamental domain of the modular group $G_{2}=\{ \pm C \in \operatorname{PSL}(2, \mathbb{Z}) ; C \equiv I \bmod 2\}$ of level 2. We will say that both $\Delta$ and $\Delta^{\prime}$ are of level 0 . A Farey triangle which 
shares a side with that of level 0 will be called of level 1 unless it is of level 0 . Similarly, a Farey triangle which shares a side with that of level $n$ will be called of level $n+1$ unless it is of level $\leq n$. It is important to note that the corresponding graph to the above tessellation is a tree, namely, there is no closed circuit.

It is well known that the orbit of 0 under the action of $\operatorname{PSL}(2, \mathbb{Z})$ coincides with $\widehat{\mathbb{Q}}$. We denote by $\tilde{\mathcal{F}}(n)$ the set of rationals which appear as vertices of Farey triangles of level $\leq n$. Set $\mathcal{F}(n)=\tilde{\mathcal{F}}(n) \backslash \tilde{\mathcal{F}}(n-1)$ for $n=0,1, \ldots$ For instance, $\mathcal{F}(0)=$ $\{-1,0,1, \infty\}, \mathcal{F}(1)=\{-2,-1 / 2,1 / 2,2\}, \mathcal{F}(2)=\{-3,-3 / 2,-2 / 3,-1 / 3,1 / 3,2 / 3$, $3 / 2,3\}$ and so on. We note that $\# \mathcal{F}(n)=2^{n+1}$ for $n \geq 1$. An element $r$ in $\mathcal{F}(n)$ will be called of level $n$ and designated by level $(r)=n$. Two rationals, $r_{1}$ and $r_{2}$, in $\widehat{\mathbb{Q}}$ are said to be Farey neighbours if $r_{1}$ and $r_{2}$ are distinct vertices of a common Farey triangle. For convenience, we adopt the notation $r_{1} \sim r_{2}$ to mean that $r_{1}$ and $r_{2}$ are Farey neighbours. It should be remembered that this is not an equivalence relation. Note that if $r_{1} \sim r_{2}$, then $\left|\operatorname{level}\left(r_{1}\right)-\operatorname{level}\left(r_{2}\right)\right| \geq 1$ unless $\operatorname{level}\left(r_{1}\right)=\operatorname{level}\left(r_{2}\right)=0$.

For two rationals $r_{1}=p_{1} / q_{1}$ and $r_{2}=p_{2} / q_{2}$ in $\widehat{\mathbb{Q}}$, where $p_{j}$ and $q_{j}$ are relatively prime integers, we assign the number $D\left(r_{1}, r_{2}\right)=\left|p_{1} q_{2}-p_{2} q_{1}\right|$. Note that this is independent of the particular choice of signs of $p_{j}$ and $q_{j}$. The following statement will be useful in our argument below.

Lemma 5.3. Two rationals $r_{1}$ and $r_{2}$ are Farey neighbours if and only if $D\left(r_{1}, r_{2}\right)$ $=1$.

Proof. First assume that $D\left(r_{1}, r_{2}\right)=1$. Then $r_{1}$ and $r_{2}$ can be written in the form $r_{1}=p_{1} / q_{1}$ and $r_{2}=p_{2} / q_{2}$ so that $p_{1} q_{2}-p_{2} q_{1}=1$. The Möbius transformation $g(z)=\left(p_{1} z+p_{2}\right) /\left(q_{1} z+q_{2}\right)$ sends $\infty$ to $r_{1}$ and 0 to $r_{2}$, which implies that the Farey triangle $g(\Delta)$ contains $r_{1}$ and $r_{2}$ as vertices. The converse can be seen similarly.

Let $r_{1}$ and $r_{2}$ be Farey neighbours with $0 \leq r_{1}<r_{2} \leq \infty$. Then they are represented in the form $r_{j}=p_{j} / q_{j}$, where $p_{j}$ and $q_{j}$ are non-negative integers for $j=1,2$ and satisfy $p_{2} q_{1}-p_{1} q_{2}=1$. We define $r_{1} \oplus r_{2}=r_{2} \oplus r_{1}=\left(p_{1}+p_{2}\right) /\left(q_{1}+q_{2}\right)$. Similarly, we define $r_{1} \ominus r_{2}=r_{2} \ominus r_{1}=\left(p_{1}-p_{2}\right) /\left(q_{1}-q_{2}\right)$. For these operations, one can show the following result.

Lemma 5.4. Let $r_{1}$ and $r_{2}$ be Farey neighbours with $0 \leq r_{1}<r_{2} \leq \infty$. Then $\Delta\left(r_{1}, r_{2}, r_{1} \oplus r_{2}\right)$ and $\Delta\left(r_{1}, r_{2}, r_{1} \ominus r_{2}\right)$ are Farey triangles sharing the side connecting $r_{1}$ with $r_{2}$ and the inequality $r_{2}<r_{1} \oplus r_{2}<r_{1}$ holds.

Conversely, any rational $r$ with $0<r<\infty$ can uniquely be decomposed into the form $r=r_{1} \oplus r_{2}$ for rationals $0 \leq r_{1}<r_{2} \leq \infty$ with $r_{1} \sim r_{2}$. Furthermore, if $r \neq 1$, then level $(r)=\max \left\{\operatorname{level}\left(r_{1}\right), \operatorname{level}\left(r_{2}\right)\right\}+1$ and $\operatorname{level}\left(r_{1}\right) \neq \operatorname{level}\left(r_{2}\right)$.

Proof. It is immediate to see that $r_{1} \oplus r_{2} \sim r_{j}$ and $r_{1} \ominus r_{2} \sim r_{j}$ for $j=1,2$ and that $r_{1}<r_{1} \oplus r_{2}<r_{2}$. Hence, the first half of the assertion has been shown. To show the second half, assume that $0<r<\infty$ is a rational and let $n=\operatorname{level}(r)$. Then there is a sequence of Farey triangles $\Delta_{0}=\Delta, \Delta_{1}, \ldots, \Delta_{n}$ such that $\Delta_{k-1}$ and $\Delta_{k}$ have a common side for $k=1, \ldots, n$ and that $\Delta_{n}$ has $r$ as its vertex. Set $\Delta_{-1}=\Delta^{\prime}$ for convenience. Note that $\Delta_{k}$ is of level $k$ and is contained in the right half of the upper half plane $\mathbb{H}$ for $k=0,1, \ldots, n$. Let $r_{1}$ and $r_{2}$ be the other two vertices of $\Delta_{n}$. Since the side of $\Delta_{n}$ connecting $r_{1}$ with $r_{2}$ separates $r$ from the left half of $\mathbb{H}, r$ lies between $r_{1}$ and $r_{2}$, and hence, the relation $r=r_{1} \oplus r_{2}$ follows. Assume now that $r \neq 1$. Then $n>0$ and $\Delta_{n-1}=\Delta\left(r_{1}, r_{2}, r_{1} \ominus r_{2}\right)$. In particular, $\max \left\{\operatorname{level}\left(r_{1}\right)\right.$, level $\left.\left(r_{2}\right)\right\} \leq n-1$. On the other hand, $\Delta_{n-1}$ shares a side 
joining $r_{1} \ominus r_{2}$ and, say, $r_{2}$ with $\Delta_{n-2}$. We see then that level $\left(r_{1}\right)=n-1$ and that $\operatorname{level}\left(r_{2}\right) \leq n-2$.

For each rational $r$ with $0<r<\infty, r \neq 1$, we define $r^{*}$ by $r^{*}=r_{1} \ominus r_{2}$, where $r_{1}$ and $r_{2}$ are as above. Then $r^{*}<\min \left\{r_{1}, r_{2}\right\}$ or $r^{*}>\max \left\{r_{1}, r_{2}\right\}$. From the above proof, one can also see that $\max \left\{\operatorname{level}\left(r^{*}\right), \operatorname{level}\left(r_{2}\right)\right\}=n-2$ if level $\left(r_{1}\right)=n-1$. Moreover, under the assumption that $r_{1}<r_{2}$, the inequality $r_{2}<r^{*}$ holds if and only if level $\left(r_{1}\right)=n-1$.

Finally, we extend the above operations for all pairs $\left(r_{1}, r_{2}\right)$ of Farey neighbours. When $0 \leq-r_{1},-r_{2} \leq \infty$, we set $r_{1} \oplus r_{2}=-\left(-r_{1}\right) \oplus\left(-r_{2}\right)$ and $r_{1} \ominus r_{2}=-\left(-r_{1}\right) \ominus$ $\left(-r_{2}\right)$ unless $\left\{r_{1}, r_{2}\right\}=\{0, \infty\}$. Also let $r^{*}=-(-r)^{*}$ for a rational $r$ with $0<$ $-r<\infty$ and $r \neq-1$. For $r= \pm 1$, we set $r^{*}=\mp 1$.

We now return to our case. Let $X$ be a marked once-punctured torus with Teichmüller parameter $\tau$. Let $H: \mathbb{R}^{2} \rightarrow \mathbb{R}^{2}=\mathbb{C}$ be the $\mathbb{R}$-linear map sending $(1,0)$ to 1 and $(0,1)$ to $\tau$. Then $H$ induces a homeomorphism $h$ from the topological oncepunctured torus $\Sigma$ onto $X=\left(\mathbb{C} \backslash L_{\tau}\right) / L_{\tau}$. The terminology above is translated, via $h: \Sigma \rightarrow X$, to that for $X$ with standard generator pair $\left(\theta_{0}, \theta_{\infty}\right)$ of the Fuchsian group $\Gamma$ uniformizing $X$. For instance, a non-peripheral simple closed curve $\gamma$ in $X$ has slope $r$ if and only if $h_{*}^{-1}(\gamma)$ has slope $r$. By Proposition 5.1 the conjugacy class of an element $\theta_{r}$ in $\Gamma$ representing a non-peripheral simple closed curve of slope $r$ is uniquely determined up to inversion. In particular, the squared trace function $\operatorname{Tr}_{\theta_{r}}^{2}: B_{2}(\mathbb{D}, \Gamma) \rightarrow \mathbb{C}$ is determined by its slope. We now explain how to compute the trace functions $\operatorname{Tr}_{\tilde{\theta}_{r}}$ for all $r \in \widehat{\mathbb{Q}}$ and suitable representatives $\tilde{\theta}_{r}$ of $\theta_{r}$ in $\mathrm{SU}(1,1)$ in a recursive way by using only the values for $r=0,1$ and $\infty$.

We begin by setting $\sigma_{r}=\operatorname{Tr}_{\tilde{\theta}_{r}}$ for $r=0,1, \infty$, where $\tilde{\theta}_{0}$ and $\tilde{\theta}_{\infty}$ are arbitrarily fixed representatives of $\theta_{0}$ and $\theta_{\infty}$, respectively, and $\tilde{\theta}_{1}=\tilde{\theta}_{0} \tilde{\theta}_{\infty}$. Define $\sigma_{-1}$ by the formula $\sigma_{-1}=\sigma_{0} \sigma_{\infty}-\sigma_{1}$. If we have defined the functions $\sigma_{r}: B_{2}(\mathbb{D}, \Gamma) \rightarrow \mathbb{C}$ for all $r \in \tilde{\mathcal{F}}(n)$, we then define $\sigma_{r}$ for $r \in \mathcal{F}(n+1)$ by the formula

$$
\sigma_{r}=\sigma_{r_{1}} \sigma_{r_{2}}-\sigma_{r^{*}}
$$

where $r_{1}$ and $r_{2}$ are Farey neighbours satisfying $r=r_{1} \oplus r_{2}$. Note here that $r_{1}, r_{2}$ and $r^{*}$ are all in $\tilde{\mathcal{F}}(n)$. In this way, we can define the functions $\sigma_{r}$ for all $r \in \widehat{\mathbb{Q}}$.

Proposition 5.5. The mapping $\sigma_{r}: B_{2}(\mathbb{D}, \Gamma) \rightarrow \mathbb{C}$ gives the trace function for a non-peripheral simple closed curve of slope $r$ in $X$ for each $r \in \widehat{\mathbb{Q}}$.

Proof. In order to prove the proposition, we construct elements $\tilde{\theta}_{r}$ in $\widetilde{\Gamma}=P^{-1}(\Gamma)$ representing non-peripheral simple closed curves of slope $r$ in a concrete way. Note that a similar construction can be found in [15]. We start with $\tilde{\theta}_{0}, \tilde{\theta}_{\infty}$ and $\tilde{\theta}_{1}=$ $\tilde{\theta}_{0} \tilde{\theta}_{\infty}$, which represents a simple closed curve of slope 1. By Corollary [5.2, $\left(\theta_{0}, \theta_{1}\right)$ and $\left(\theta_{1}, \theta_{\infty}\right)$ are standard generator pairs of $\Gamma$, where $\theta_{1}=P\left(\tilde{\theta}_{1}\right)$. Suppose that we have defined $\tilde{\theta}_{r} \in \widetilde{\Gamma}$ for all $r \in \tilde{\mathcal{F}}_{+}(n):=\tilde{\mathcal{F}}(n) \cap[0, \infty]$ in such a way that the following properties are satisfied:

(1) $\theta_{r}$ represents a simple closed curve of slope $r$ in $X$ for $r \in \tilde{\mathcal{F}}_{+}(n)$, where $\theta_{r}=P\left(\tilde{\theta}_{r}\right)$, and

(2) $\left(\theta_{r_{1}}, \theta_{r_{2}}\right)$ is a standard generator pair for each pair of Farey neighbours $r_{1}, r_{2} \in \tilde{\mathcal{F}}_{+}(n)$ with $0 \leq r_{1}<r_{2} \leq \infty$. 
Then, for $r \in \mathcal{F}(n+1)$ with $0<r<\infty$, we define $\tilde{\theta}_{r}$ by

$$
\tilde{\theta}_{r}=\tilde{\theta}_{r_{1}} \tilde{\theta}_{r_{2}}
$$

where $r_{1}$ and $r_{2}$ are unique Farey neighbours with $0 \leq r_{1}<r_{2} \leq \infty$ satisfying $r=r_{1} \oplus r_{2}$ (see Lemma 5.4). Note also, by Corollary 5.2, that $\left(\theta_{r_{1}}, \theta_{r}\right)$ and $\left(\theta_{r}, \theta_{r_{2}}\right)$ are standard generator pairs of $\Gamma$, where $\theta_{r}=P\left(\tilde{\theta}_{r}\right)$. In particular, $\theta_{r}$ represents a simple closed curve with slope $r$. In this way, we extend $\tilde{\theta}_{r}$ for all $r \in \tilde{\mathcal{F}}_{+}(n+1)$, while preserving the above properties (1) and (2). By induction, we extend $\tilde{\theta}_{r}$ for all $r \in \widehat{\mathbb{Q}} \cap[0, \infty]$.

For $r \in \widehat{\mathbb{Q}} \cap(-\infty, 0)$, we can apply the same argument as above by replacing $\left(\theta_{0}, \theta_{\infty}\right)$ by $\left(\theta_{\infty}, \theta_{0}^{-1}\right)$. More precisely, we set $\tilde{\theta}_{0}^{\prime}=\tilde{\theta}_{\infty}, \tilde{\theta}_{\infty}^{\prime}=\tilde{\theta}_{0}^{-1}$ and $\tilde{\theta}_{1}^{\prime}=\tilde{\theta}_{0}^{\prime} \tilde{\theta}_{\infty}^{\prime}=$ $\tilde{\theta}_{\infty} \tilde{\theta}_{0}^{-1}$. Then $\tilde{\theta}_{1}^{\prime}$ represents a simple closed curve of slope -1 in $X$. Also, in the same way as above, we let

$$
\tilde{\theta}_{r}^{\prime}=\tilde{\theta}_{r_{1}}^{\prime} \tilde{\theta}_{r_{2}}^{\prime}
$$

for $r \in \mathcal{F}(n+1)$ with $0<r<\infty$ after defining $\tilde{\theta}_{s}^{\prime}$ for all $s \in \tilde{\mathcal{F}}_{+}(n)$. Finally, we set $\tilde{\theta}_{-r}=\tilde{\theta}_{r}^{\prime}$ for $r \in \widehat{\mathbb{Q}} \cap(-\infty, 0)$. Then we can easily see that $\theta_{-r}=P\left(\tilde{\theta}_{-r}\right)$ represents a simple closed curve of slope $-r$ in $X$.

We are now at the final stage to show the assertion. The key is the general formula

$$
\operatorname{tr} A B+\operatorname{tr} A^{-1} B=\operatorname{tr} A \operatorname{tr} B
$$

for $A, B \in \operatorname{SL}(2, \mathbb{C})$. Let $\hat{\sigma}_{r}=\operatorname{Tr}_{\tilde{\theta}_{r}}$ for $r \in \widehat{\mathbb{Q}}$. Then $\hat{\sigma}_{r}=\sigma_{r}$ for all $r \in \tilde{\mathcal{F}}(0)$. We show the same statement for all $r \in \tilde{\mathcal{F}}(n)$ for each $n$ by induction. Suppose that we have shown this up to $n$. Let $r \in \mathcal{F}(n+1)$ and $r_{1}$ and $r_{2}$ be unique Farey neighbours with $r_{1}<r_{2}$ satisfying $r=r_{1} \oplus r_{2}$. Since $r_{1}, r_{2}, r^{*}=r_{1} \ominus r_{2} \in \tilde{\mathcal{F}}(n)$, we see that $\hat{\sigma}_{s}=\sigma_{s}$ for $s=r_{1}, r_{2}, r^{*}$. As we observed above, $r_{2}=r_{1} \oplus r^{*}$ if $r_{2}<r^{*}$. In this case, $\tilde{\theta}_{r^{*}}=\tilde{\theta}_{r_{1}}^{-1} \tilde{\theta}_{r_{2}}$, and hence,

$$
\begin{aligned}
\hat{\sigma}_{r}(\varphi) & =\operatorname{tr} \tilde{\chi}_{\varphi}\left(\tilde{\theta}_{r_{1}} \tilde{\theta}_{r_{2}}\right)=\operatorname{tr} \tilde{\chi}_{\varphi}\left(\tilde{\theta}_{r_{1}}\right) \operatorname{tr} \tilde{\chi}_{\varphi}\left(\tilde{\theta}_{r_{2}}\right)-\operatorname{tr} \tilde{\chi}_{\varphi}\left(\tilde{\theta}_{r_{1}}^{-1} \tilde{\theta}_{r_{2}}\right) \\
& =\sigma_{r_{1}}(\varphi) \sigma_{r_{2}}(\varphi)-\sigma_{r^{*}}(\varphi)=\sigma_{r}(\varphi) .
\end{aligned}
$$

When $r^{*}<r_{1}$, the relation $r_{1}=r^{*} \oplus r_{2}$ holds, and hence, $\tilde{\theta}_{r^{*}}=\tilde{\theta}_{r_{1}} \tilde{\theta}_{r_{2}}^{-1}$. Since $\operatorname{tr} A^{-1} B=\operatorname{tr} A B^{-1}$, we can see the same relation $\hat{\sigma}_{r}=\sigma_{r}$ as above. We now conclude that $\operatorname{Tr}_{\tilde{\theta}_{r}}=\sigma_{r}$ for all $r \in \widehat{\mathbb{Q}}$ by induction.

\section{McMullen's Bending Coordinates of the Bers Slice}

Following McMullen [26], we describe the bending coordinates of the Teichmüller space $T(\Gamma)$ of a once-punctured torus $X=\mathbb{D} / \Gamma$. For precise definitions for the terminology below, see 26 or references cited there.

Considering the slope, we have identified the space of simple closed geodesics on $X$ with $\widehat{\mathbb{Q}}=\mathbb{Q} \cup\{\infty\}$. Extending this continuously, we obtain the homeomorphic identification of the projectivized space $\mathbb{P} \mathcal{M L}(X)$ of measured laminations on $X$ with the extended real line $\widehat{\mathbb{R}} \cong \mathrm{S}^{1}$.

Let $\varphi \in T(X) \backslash\{0\}$ be a point in the Bers embedding. The group $\chi_{\varphi}(\Gamma)$ is then quasi-Fuchsian and its region of discontinuity consists of two quasidisks $f_{\varphi}(\mathbb{D})$ and $\widehat{\mathbb{C}} \backslash \overline{f_{\varphi}(\mathbb{D})}$, which are denoted by $\Omega_{\varphi}^{+}$and $\Omega_{\varphi}^{-}$, respectively. We remark that $\chi_{\varphi}(\Gamma)$ is never Fuchsian for $\varphi \in T(\Gamma) \backslash\{0\}$. Since $f_{\varphi}$ is conformal on $\mathbb{D}, \Omega_{\varphi}^{+} / \chi_{\varphi}(\Gamma)$ 
is always conformally equivalent to $X$, whereas $X_{\varphi}^{*}:=\Omega_{\varphi}^{-} / \chi_{\varphi}(\Gamma)$ varies. We denote by $\mathcal{C}_{\varphi}$ the hyperbolic convex hull of the limit set $\Lambda_{\varphi} \subset \widehat{\mathbb{C}}$ of $\chi_{\varphi}(\Gamma)$ in the hyperbolic three-space $\mathbb{H}^{3}$, where we identify $\widehat{\mathbb{C}}$ with the sphere at infinity of $\mathbb{H}^{3}$. Then the boundary $\partial \mathcal{C}_{\varphi}$ in $\mathbb{H}^{3}$ consists of two connected components $\partial \mathcal{C}_{\varphi}^{ \pm}$facing to $\Omega_{\varphi}^{ \pm}$. We write $M_{\varphi}$ for the hyperbolic three manifold $\mathbb{H}^{3} / \chi_{\varphi}(\Gamma)$. Then $\mathcal{C}_{\varphi} / \chi_{\varphi}(\Gamma)$ is the convex core of $M_{\varphi}$, whose boundary consists of two connected components $\partial \mathcal{C}_{\varphi}^{ \pm} / \chi_{\varphi}(\Gamma)$. The nearest point retraction from $\Omega_{\varphi}^{ \pm}$to $\partial \mathcal{C}_{\varphi}^{ \pm}$induces the homotopy equivalence between $\Omega_{\varphi}^{ \pm} / \chi_{\varphi}(\Gamma)$ and $\partial \mathcal{C}_{\varphi}^{ \pm} / \chi_{\varphi}(\Gamma)$, and hence, $\partial \mathcal{C}_{\varphi}^{ \pm} / \chi_{\varphi}(\Gamma)$ are both topological punctured tori (see for example [5]). The surfaces $\partial \mathcal{C}_{\varphi}^{ \pm} / \chi_{\varphi}(\Gamma)$ are endowed with the hyperbolic metric by $M_{\varphi}$ and both are pleated surfaces with the natural bending measures on their pleating loci. We denote by $\left[\mathrm{pl}^{ \pm}(\varphi)\right]$ the projective class in $\mathbb{P} \mathcal{M L}(X)$ of the pleating locus of $\mathcal{C}_{\varphi}^{ \pm} / \chi_{\varphi}(\Gamma)$. Note that each connected component of $\partial \mathcal{C}_{\varphi}^{ \pm}$minus its pleating locus is contained in a hyperbolic plane in $\mathbb{H}^{3}$. We write $\ell_{\mathrm{pl}^{-}(\varphi)}\left(M_{\varphi}\right)$ and $\ell_{\mathrm{pl}^{-}(\varphi)}(X)$ for the hyperbolic length of the bending lamination $\mathrm{pl}^{-}(\varphi)$ on $\partial \mathcal{C}_{\varphi}^{-} / \chi_{\varphi}(\Gamma)$ and on $X$, respectively. Now we can state a result of McMullen [26] on the bending coordinates of $T(\Gamma)$.

Theorem C ([26, Theorem 1.5]). The mapping

$$
\Phi: \varphi \mapsto\left(\left[\mathrm{pl}^{-}(\varphi)\right], \frac{\ell_{\mathrm{pl}^{-}(\varphi)}\left(M_{\varphi}\right)}{\ell_{\mathrm{pl}^{-}(\varphi)}(X)}\right)
$$

gives a homeomorphism from $T(\Gamma) \backslash\{0\}$ onto $\mathbb{P} \mathcal{M L}(X) \times(0,1)=\widehat{\mathbb{R}} \times(0,1)$, where we identify $\mathbb{P} \mathcal{M L}(X)$ with $\widehat{\mathbb{R}} \cong \mathrm{S}^{1}$ as above.

For $r \in \widehat{\mathbb{R}}$, we set $\mathcal{P}_{r}=\Phi^{-1}(\{r\} \times(0,1))$. These proper arcs in $T(\Gamma) \backslash\{0\}$ will be called pleating rays. In the special case where for $r \in \widehat{\mathbb{Q}}, \mathcal{P}_{r}$ is called a rational pleating ray of slope $r$. We remark that $\ell_{\mathrm{pl}^{-}(\varphi)}(X)$ is constant on $\mathcal{P}_{r}$. In particular, if $r \in \widehat{\mathbb{Q}}$, we have $\ell_{\mathrm{pl}^{-}(\varphi)}(X)=\operatorname{arccosh}\left(\left|\operatorname{tr} \theta_{r}\right| / 2\right)$ for $\varphi \in \mathcal{P}_{r}$.

The next result guarantees the computability of the rational pleating rays up to the accessory parameter. For $r \in \widehat{\mathbb{Q}}$, put

$\mathcal{H}_{r}:=\left\{\varphi \in B_{2}(\mathbb{D}, \Gamma) ; \sigma_{r}(\varphi)\right.$ is real and $\left.\sigma_{r}(\varphi)^{2}>4\right\}=\sigma_{r}^{-1}((-\infty,-2) \cup(2,+\infty))$ and call it the hyperbolic locus of slope $r$. Note that $0 \in \mathcal{H}_{r}$ for all $r \in \widehat{\mathbb{Q}}$.

Theorem 6.1. The rational pleating ray $\mathcal{P}_{r}, r \in \widehat{\mathbb{Q}}$, is the unique connected component of $\mathcal{H}_{r} \backslash\{0\}$ with endpoint 0 on which $\sigma_{r}(\varphi)^{2}$ is strictly smaller than $\sigma_{r}(0)^{2}$.

Besides Theorem $\mathrm{C}$, we need the local pleating theorem due to Keen and Series [14, Theorem 8.1] for the proof of our theorem. We state it here in a specialized form for our aim.

Theorem D. Let $\varphi_{0} \in \mathcal{P}_{r} \cup\{0\}$ for some $r \in \widehat{\mathbb{Q}}$. Then there exists a neighbourhood $U$ of $\varphi_{0}$ in $T(\Gamma)$ such that every element $\varphi \in\left(\mathcal{H}_{r} \backslash\{0\}\right) \cap U$ satisfies either $\left[\mathrm{pl}^{-}(\varphi)\right]=$ $r$ or $\left[\mathrm{pl}^{+}(\varphi)\right]=r$.

Proof of Theorem [6.1. Let $r \in \widehat{\mathbb{Q}}$. We may assume that $\sigma_{r}(0)>2$. Take a point $\varphi \in \mathcal{P}_{r}$, then the axis of $\chi_{\varphi}\left(\theta_{r}\right)$ in $\mathbb{H}^{3}$ lies on the boundary $\partial \mathcal{C}_{\varphi}^{-}$which is invariant under $\chi_{\varphi}\left(\theta_{r}\right)$. Therefore $\chi_{\varphi}\left(\theta_{r}\right)$ does not rotate $\mathbb{H}^{3}$ around the axis; namely, it is hyperbolic (see [14, Proposition 6.4] for details) which implies that $\mathcal{P}_{r}$ is contained in $\mathcal{H}_{r} \backslash\{0\}$. 


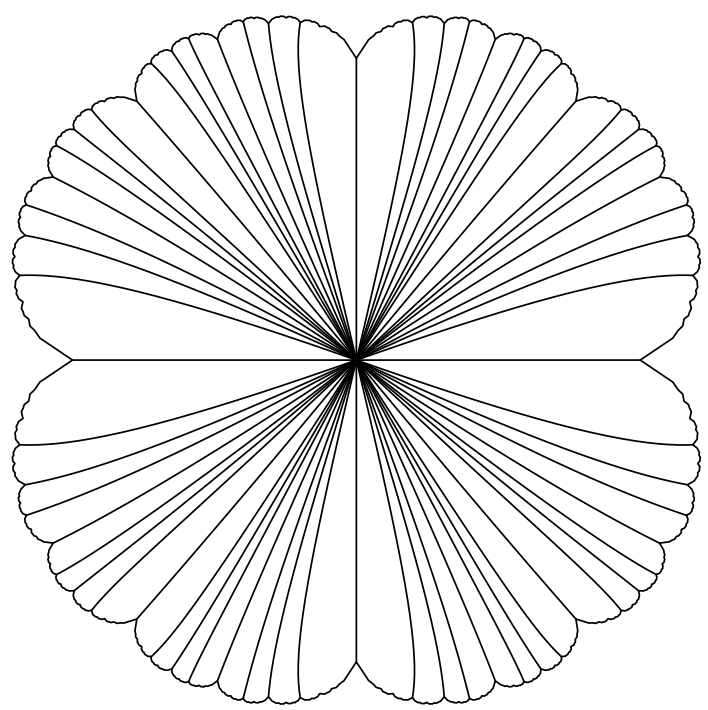

Figure 1. Bers embedding of the once-punctured square torus with pleating rays of level $\leq 4$

Recall the inequalities due to McMullen [26, Corollary 3.5]

$$
\begin{gathered}
\ell_{\mathrm{pl}^{-}(\varphi)}\left(X_{\varphi}^{*}\right)<\ell_{\mathrm{pl}^{-}(\varphi)}\left(M_{\varphi}\right)<\ell_{\mathrm{pl}^{-}(\varphi)}(X) \text { and } \\
\ell_{\mathrm{pl}^{+}(\varphi)}(X)<\ell_{\mathrm{pl}^{+}(\varphi)}\left(M_{\varphi}\right)<\ell_{\mathrm{pl}^{+}(\varphi)}\left(X_{\varphi}^{*}\right)
\end{gathered}
$$

for every $\varphi \in T(\Gamma) \backslash\{0\}$. Since $\ell_{\mathrm{pl}^{-}(\varphi)}\left(M_{\varphi}\right)=\operatorname{arccosh}\left(\sigma_{r}(\varphi) / 2\right)$ for $\varphi \in \mathcal{P}_{r}$, we see that $\sigma_{r}(\varphi) \rightarrow 2$ as $\varphi$ approaches the Bers boundary along $\mathcal{P}_{r}$ by Theorem $\mathbb{C}$ This means that the endpoint of $\mathcal{P}_{r}$ corresponds to the cusp pinched along the simple closed geodesic of slope $r$. In particular, $\mathcal{P}_{r}$ is closed in $\mathcal{H}_{r} \backslash\{0\}$.

In order to show that $\mathcal{P}_{r}$ is a connected component of $\mathcal{H}_{r} \backslash\{0\}$ ending at the basepoint 0 on which $\sigma_{r}<\sigma_{r}(0)$, it suffices to see that $\mathcal{P}_{r}$ is open in $\mathcal{H}_{r} \backslash\{0\}$. Suppose that $\varphi_{0} \in \mathcal{P}_{r}$. Note from (6.1) that $2<\sigma_{r}\left(\varphi_{0}\right)<\sigma_{r}(0)$. Therefore, $\left|\sigma_{r}\right|<$ $\sigma_{r}(0)$ in a sufficiently small neighbourhood $U$ of $\varphi_{0}$ in $T(\Gamma) \backslash\{0\}$. By Theorem D, we can further choose $U$ so that every $\varphi \in \mathcal{H}_{r} \cap U$ satisfies $\left[\mathrm{pl}^{-}(\varphi)\right]=r$ or $\left[\mathrm{pl}^{+}(\varphi)\right]=r$. If the latter case occurred, (6.2) would yield $\sigma_{r}(0)<\left|\sigma_{r}(\varphi)\right|$. However, this contradicts the choice of $U$. Therefore, only the first case occurs, namely, $\mathcal{H}_{r} \cap$ $U \subset \mathcal{P}_{r}$. Thus the openness follows. The uniqueness immediately follows from Theorems [C] and D.

We emphasize that the set described in the above theorem can be computed, at least in a numerical way, by tracing the real locus of the function $\sigma_{r}$ in the decreasing direction from the origin. Figures 1 and 2 were created in this way. The above theorem shows also that the entire function $\sigma_{r}$ has no branch point on the pleating ray $\mathcal{P}_{r}$ and at the origin. Furthermore, H. Miyachi [28] recently proved that $\sigma_{r}$ has no branch point at the cusp corresponding to $\theta_{r}$. Summarizing the above, we obtain the next result.

Corollary 6.2. Each rational pleating ray $\mathcal{P}_{r}$ in $T(\Gamma)$ with its endpoints is a regular analytic simple closed arc and $\sigma_{r}(\varphi)^{2}$ is positive real and decreases to 4 when $\varphi$ 


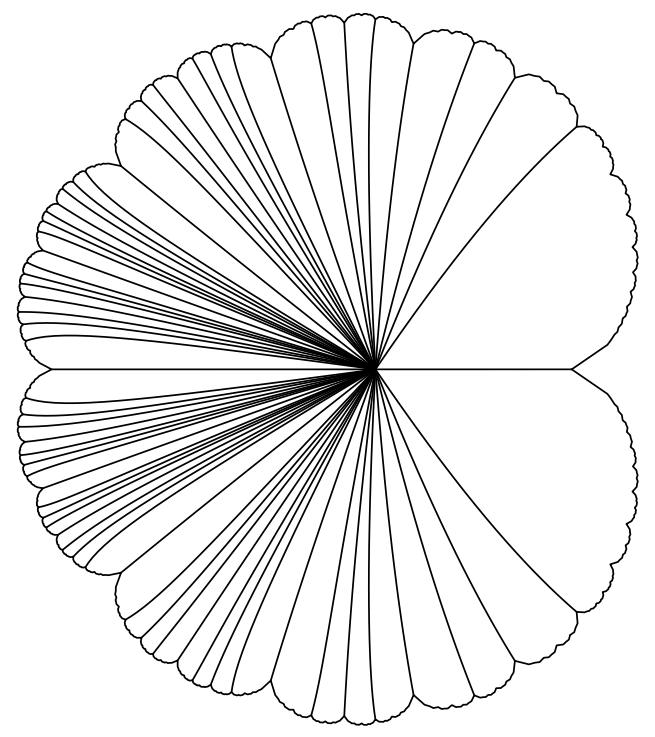

Figure 2. Bers embedding of a once-punctured "long" rectangular torus $(\lambda=0.001)$

moves along the ray toward the cusp on the Bers boundary of $T(\Gamma)$ corresponding to slope $r$.

We take a closer look at the analytic structure of pleating rays for the later use.

Theorem 6.3. Any two rational pleating rays intersect transversally at the basepoint unless the tangent vectors have opposite directions there.

The above assertion seems true even when we allow all pleating rays. We, however, content ourselves here with the above form since we will require only the case of rational pleating rays in this paper. See the final part of this section explaining how to numerically compute the value of the accessory parameter and a standard pair of generators of a Fuchsian group.

We should comment on the similar result for the Maskit embedding of the Teichmüller space of once-punctured tori (see [15, [27] and [40]). The Maskit embedding is a deformation space of terminal regular $b$-groups of type $(1,1)$ and it is realized as an unbounded domain in $\mathbb{H}$, where $\infty$ plays the same role as the basepoint in the Bers embedding even though no groups correspond to $\infty$. Rational pleating rays can also be considered for the Maskit embedding; among other things, Keen and Series [15, Proposition 3.2] showed that for a point $\mu=s+i t$ of the rational pleating ray of slope $p / q$, the real part $s$ tends to $2 p / q$ as $t \rightarrow \infty$. This result implies that the asymptotic behaviour of rational pleating rays at the basepoint ( $\infty$ for the Maskit embedding) characterizes them.

To prove Theorem 6.3 we need a preliminary result connecting the real analytic structure of the Teichmüller space with the complex analytic one, which might be of independent interest.

Let $\gamma$ be a hyperbolic element in an arbitrarily given Fuchsian group $\Gamma$. We denote by $\ell_{\gamma}: T(\Gamma) \rightarrow \mathbb{R}_{+}$the length function on $T(\Gamma)$ corresponding to $\gamma$; namely, 
for $\varphi \in T(\Gamma), \ell_{\gamma}(\varphi)$ is the hyperbolic length of the closed geodesic corresponding to $\gamma$ in the Riemann surface $\Omega_{\varphi}^{-} / \chi_{\varphi}(\Gamma)$, where $\Omega_{\varphi}^{-}=\widehat{\mathbb{C}} \backslash \overline{f_{\varphi}(\mathbb{D})}$. Let $\varepsilon_{\gamma}(\varphi)$ be the logarithm of the multiplier of $\chi_{\varphi}(\gamma)$ with positive real part. Therefore, $\sigma_{\gamma}^{2}(\varphi)=$ $\operatorname{tr}^{2} \chi_{\varphi}(\gamma)=2 \cosh \left(\varepsilon_{\gamma}(\varphi)\right)+2$ and $\operatorname{Re} \varepsilon_{\gamma}(\varphi)>0$. If we knew the conformal mapping $g_{\varphi}$ from $\mathbb{D}$ onto $\Omega_{\varphi}^{-}$, we could relate $\ell_{\gamma}$ to $\varepsilon_{\gamma}$ by the formula

$$
\operatorname{tr}^{2}\left[g_{\varphi}{ }^{-1} \circ \chi_{\varphi}(\gamma) \circ g_{\varphi}\right]=2 \cosh \ell_{\gamma}(\varphi)+2 .
$$

It is, however, difficult to find $g_{\varphi}$, in general. We can, at least, say something regarding its origin.

Lemma 6.4. For a fixed $\varphi \in B_{2}(\mathbb{D}, \Gamma)$, the formula

$$
\left.\frac{d}{d t} \ell_{\gamma}(t \varphi)\right|_{t=0}=\left.\operatorname{Re} \frac{d}{d t} \varepsilon_{\gamma}(t \varphi)\right|_{t=0}
$$

holds, where the differentiation is taken with respect to the real parameter $t$.

Proof. We refer the reader to [10] and [29] as standard textbooks for the basic facts used herein. In this proof, we take advantage of the lower half plane $\mathbb{H}^{*}$ instead of the unit disk $\mathbb{D}$, so that we regard $\Gamma$ as a Fuchsian group acting on $\mathbb{H}$. This procedure is harmlessly done, as usual, through the Cayley transform $z \mapsto-i(1+z) /(1-z)$.

Let $\mu$ be a Beltrami differential for $\Gamma$ in $\mathbb{H}$ with $\|\mu\|_{\infty}<1$. We extend $\mu$ to be 0 in $\mathbb{H}^{*}$. On the other hand, let $\mu^{*}$ denote the Beltrami coefficient for $\Gamma$ defined by

$$
\mu^{*}(z)= \begin{cases}\mu(z), & z \in \mathbb{H}, \\ \overline{\mu(\bar{z})}, & z \in \mathbb{H}^{*} .\end{cases}
$$

We denote by $F^{\mu}$ and $F_{\mu}$ the quasiconformal self-homeomorphism of $\mathbb{C}$ with Beltrami coefficients $\mu$ and $\mu^{*}$, respectively, normalized so as to fix 0 and 1 . Note that $F_{\mu}$ maps $\mathbb{H}$ onto itself while $F^{\mu}$ maps $\mathbb{H}^{*}$ conformally onto the quasidisk $F^{\mu}\left(\mathbb{H}^{*}\right)$. (Note also that $F^{\mu} \circ F_{\mu}^{-1}$ is a conformal mapping from $\mathbb{H}$ onto $F^{\mu}(\mathbb{H})$.) Therefore, $F_{\mu} \Gamma F_{\mu}{ }^{-1}$ is a Fuchsian group while $F^{\mu} \Gamma\left(F^{\mu}\right)^{-1}$ is a Kleinian group acting on

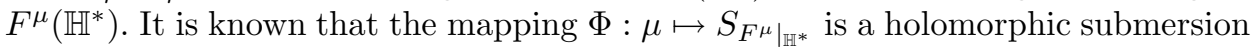
from the open unit ball of the Banach space of Beltrami differentials for $\Gamma$ onto $T(\Gamma)$.

For a given $\varphi$, we take a Beltrami differential $\mu$ in such a way that the Fréchet derivative of $\Phi$ at the origin sends $\mu$ to $\varphi$.

Let $\gamma \in \Gamma$ be a hyperbolic element. Conjugating by a suitable Möbius transformation, we may assume that $\gamma$ is expressed in the form $\gamma(z)=e^{\varepsilon_{0}} z$, where $\varepsilon_{0}>0$ is a constant. Set $\gamma^{t}=F^{t \mu} \circ \gamma \circ\left(F^{t \mu}\right)^{-1}$ and $\gamma_{t}=F_{t \mu} \circ \gamma \circ F_{t \mu}^{-1}$ for $t \in \mathbb{R}$ small enough. Then, by definition, these Möbius transformations can be written as

$$
\gamma^{t}(z)=e^{\varepsilon(t)} z \text { and } \gamma_{t}(z)=e^{\varepsilon^{*}(t)} z .
$$

We now recall Gardiner's variation formula for the multiplier of a hyperbolic Möbius transformation under quasiconformal mappings, [6]. As Miyachi remarked in [28, $\S 8.1]$, the formula is valid not only for Fuchsian groups but also for Kleinian groups. 
Gardiner's formula reads as

$$
\begin{aligned}
\left.\frac{d}{d t} \varepsilon(t)\right|_{t=0} & =\iint_{A_{+}} \frac{\mu(z)}{z^{2}} d x d y, \quad \text { and } \\
\left.\frac{d}{d t} \varepsilon^{*}(t)\right|_{t=0} & =\iint_{A} \frac{\mu^{*}(z)}{z^{2}} d x d y=\iint_{A_{+}}\left(\frac{\mu(z)}{z^{2}}+\frac{\overline{\mu(z)}}{\bar{z}^{2}}\right) d x d y,
\end{aligned}
$$

where $A=\left\{1<|z|<e^{\varepsilon}\right\}$ and $A_{+}=A \cap \mathbb{H}$. Hence, we obtain $\left(\varepsilon^{*}\right)^{\prime}(0)=2 \operatorname{Re} \varepsilon^{\prime}(0)$.

From the definitions, the basic relations $2 \ell_{\gamma}(\Phi(t \mu))=\varepsilon^{*}(t)$ and $\varepsilon_{\gamma}(\Phi(t \mu))=\varepsilon(t)$ follow. Noting that $\Phi(t \mu)=t \varphi+O\left(|t|^{2}\right)$ as $t \rightarrow 0$, we get the desired relation between $\ell_{\gamma}$ and $\varepsilon_{\gamma}$.

Proof of Theorem 6.3. The argument and notation herein will be based on a paper of McMullen, [26]. Let $r \in \widehat{\mathbb{Q}}$. Since $\mathcal{P}_{r}$ is a real locus of an analytic function defined in $\mathbb{C}$, the unit direction vector of $\mathcal{P}_{r}$ at the origin,

$$
\Theta_{r}=\lim _{\varphi \rightarrow 0 \text { in } \mathcal{P}_{r}}\|\varphi\|_{\mathbb{D}}^{-1} \varphi
$$

is well defined. We show that the mapping $r \mapsto \Theta_{r}$ is injective on $\widehat{\mathbb{Q}}$.

First we observe that $\Theta_{r}$ is parallel to the tangent vector $\frac{d}{d t} \operatorname{gr}_{t \gamma}\left(X^{*}\right)$ at $t=$ 0 , where $\operatorname{gr}_{t \gamma}\left(X^{*}\right)$ is the point in $T(\Gamma)$ corresponding to the time $t$ grafting of the mirror image $X^{*}=X_{0}^{*}$ of $X$ along the simple closed geodesic representing a hyperbolic element $\gamma \in \Gamma$ of slope $r$. Let $u$ be the real part of the holomorphic function $\varepsilon_{\gamma}: T(\Gamma) \rightarrow \mathbb{C}$ given above. By the relation $\sigma_{\gamma}^{2}(\varphi)=2 \cosh \left(\varepsilon_{\gamma}(\varphi)\right)+2$, the vector $\Theta_{r}$ has the opposite direction to the gradient $\nabla u$ at the origin because $u$ is harmonic. On the other hand, McMullen's theorem [26. Theorem 3.8] states the formula

$$
\frac{d}{d t} \operatorname{gr}_{t \gamma}\left(X^{*}\right)=-\nabla \ell_{\gamma}\left(X^{*}\right)
$$

where the gradient is taken with respect to the Weil-Petersson metric. In our case, the Teichmüller space $T(\Gamma)$ is one-dimensional, and therefore, the Weil-Petersson (Kähler) metric is conformally equivalent to the Euclidean metric. Now, the formula $\nabla \ell_{\gamma}=\nabla u$ deduced from Lemma 6.4 connects these facts to conclude the above claim.

To show the injectivity, we next use the fact that grafting and twisting along $\gamma$ are related by the relation $\frac{d}{d t} \operatorname{gr}_{t \gamma}\left(X^{*}\right)=i \cdot \frac{d}{d t} \operatorname{tw}_{t \gamma}\left(X^{*}\right)$ at the origin with a suitable orientation for $\gamma$ (see the proof of Theorem 3.8 in [26]). Therefore, the (positive) earthquake path and the pleating locus (with respect to $\gamma$ ) form a right angle at the basepoint. Hence, it suffices to show that different earthquake paths starting from the basepoint have different directions. This assertion has been proved by Kerckhoff [16] Theorem 3.5] in a more general setting.

We conclude this section with a comment on the numerical computation of the accessory parameter and the Fuchsian group uniformizing a given once-punctured torus.

In the above, we have assumed that we know the value of the accessory parameter. However, in practical calculations, we need to know it in advance. We now indicate how to do that by using our methods developed above. Let $\lambda \in \mathbb{C} \backslash\{0,1\}$ 
be given. Instead of (4.5), we consider the differential equation

$$
2 y^{\prime \prime}+\left\{\frac{1}{2 z^{2}(z-1)^{2}}+\frac{1}{2(z-\lambda)^{2}}+\frac{t}{z(z-1)(z-\lambda)}\right\} y=0 .
$$

We want to find the value $t=c(\lambda)$ for the given $\lambda$. Let $\alpha_{0}$ and $\alpha_{\infty}$ be the same as in Section 4 and let $\hat{L}_{t}\left[\alpha_{r}\right]$ be the transition matrix of (6.31) along the path $\alpha_{r}$ for $r=0, \infty$. We set $A(t)=\hat{L}_{t}\left[\alpha_{0}\right] M_{z_{0}}\left(A_{0}\right)$ and $B(t)=\hat{L}_{t}\left[\alpha_{\infty}\right] M_{z_{0}}\left(A_{\infty}\right)$, where $M_{z_{0}}\left(A_{r}\right)$ is given in Section 4. Then $F_{0}(t)=\operatorname{tr} A(t), F_{\infty}(t)=\operatorname{tr} B(t)$ and $F_{1}(t)=\operatorname{tr} A(t) B(t)$ are all entire functions. Let $\Gamma(t)$ be the Möbius group generated by $A(t)$ and $B(t)$. Theorem 4.3 implies that $\Gamma(c(\lambda))$ is Möbius conjugate to the original Fuchsian group $\Gamma$ uniformizing the once-punctured torus $X$ corresponding to $\tau$ where $\lambda=\lambda(\tau)$. Therefore, if $t=c(\lambda)$, the following hold:

$$
F_{0}(t), F_{\infty}(t), F_{1}(t) \in(-\infty,-2) \cup(2,+\infty) .
$$

Conversely, if $t \in \mathbb{C}$ satisfies (6.4), $t$ is a candidate for the value of the accessory parameter $c(\lambda)$. In fact, for $t$ with $(t-c(\lambda)) \psi_{0} \in T(Y), \Gamma(t)$ is never Fuchsian unless $t=c(\lambda)$. Recalling the fact that $\Gamma(t)$ is conjugate to a subgroup of $\operatorname{PSL}(2, \mathbb{R})$ precisely when the values $F_{0}(t), F_{\infty}(t)$ and $F_{1}(t)$ are all real, we see that $\Gamma(t)$ is Fuchsian if and only if (6.4) hold under the assumption that $(t-c(\lambda)) \psi_{0} \in T(Y)$. Note also that $|t-c(\lambda)|<2 /\left\|\psi_{0}\right\|_{Y}$ implies $(t-c(\lambda)) \psi_{0} \in T(Y)$ by the Ahlfors-Weill theorem (see Section 2). Therefore, we can conclude that $t=c(\lambda)$ from condition (6.4) if we are certain that $|t-c(\lambda)|<2 /\left\|\psi_{0}\right\|_{Y}$.

Hence, if we are given an initial point $t_{0}$ which is sufficiently close to $c(\lambda)$, we can construct a sequence $t_{n}$ tending to the value $c(\lambda)$ as follows. By Theorem 6.3 we can choose two of $F_{0}, F_{\infty}, F_{1}$, say $F_{0}$ and $F_{1}$, so that the corresponding pleating rays $\mathcal{P}_{0}$ and $\mathcal{P}_{1}$ are transversal at the basepoint. Then the point $t=c(\lambda)$ will be determined (at least locally) as the intersection of the real loci of functions $F_{0}$ and $F_{1}$. Set

$$
t_{2 j+1}=t_{2 j}-i \frac{\operatorname{Im} F_{0}\left(t_{2 j}\right)}{F_{0}^{\prime}\left(t_{2 j}\right)}
$$

and

$$
t_{2 j+2}=t_{2 j+1}-i \frac{\operatorname{Im} F_{1}\left(t_{2 j+1}\right)}{F_{1}^{\prime}\left(t_{2 j+1}\right)}
$$

for $j=0,1, \ldots$ Then, in a similar way to Newton's method, the sequence $t_{n}$ converges to $c(\lambda)$ if $t_{0}$ is sufficiently close to $c(\lambda)$ (see, for example, [31, $\left.\S 3.5 .2\right]$ ). Note here that the transversality of the pleating rays guarantees the convergence of $t_{n}$.

We make a few technical remarks. Since it is difficult to calculate the derivative of $F_{r}$ practically, we replace it by a suitable difference quotient in the above formulae like the Secant method. It is typical to use $\left(F_{r}\left(t_{n}\right)-F_{r}\left(t_{n-2}\right)\right) /\left(t_{n}-t_{n-2}\right)$ as the difference quotient provided that $t_{n}-t_{n-2}$ is sufficiently small.

It is difficult to give the initial point $t_{0}$ a priori for a given $\lambda$. However, if we know the value of $c\left(\lambda_{0}\right)$ for some $\lambda_{0}$ (e.g., $\lambda_{0}=1 / 2$ ), we may choose a finite sequence $\lambda_{1}, \lambda_{2}, \ldots, \lambda_{n}=\lambda$ so that $\lambda_{j}$ and $\lambda_{j-1}$ are close enough for $j=1, \ldots, n$. Then, we could compute $c\left(\lambda_{j}\right)$ by using the value of $c\left(\lambda_{j-1}\right)$ as the initial point $t_{0}$ for $\lambda_{j}$. In this way, we could reach $\lambda$ after $n$-times of this procedure. 


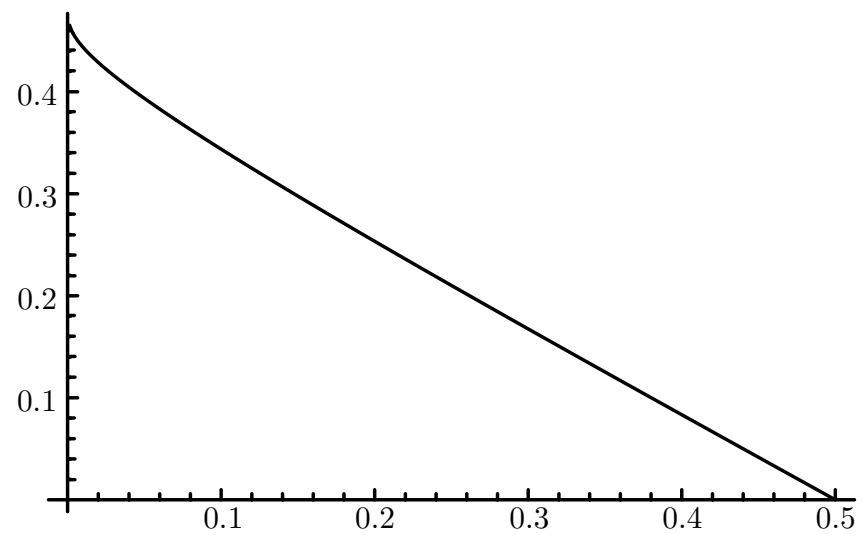

FiguRE 3. Graph of the accessory parameter $c(\lambda)$ in $0<\lambda<1 / 2$

Using the above method, we have drawn the graph of the function $c(\lambda)$ in $0<$ $\lambda<1 / 2$ in Figure 3, Note that Hempel [8] has obtained the asymptotic formula

$$
c(\lambda)=\frac{1}{2}-\frac{\pi^{2}}{2(-\log |\lambda|)^{2}}(1+o(1))
$$

as $\lambda \rightarrow 0$ in $\mathbb{C}$.

\section{Relationship with Heun's Differential equation}

In this section, we explain that our differential equation (4.5) can be translated to Heun's differential equation in the standard way. It may be more advantageous to use the latter form because that is more widely studied and the behaviour of coefficients is tamer than the former near the singularities (see [33] and its extensive references).

Heun's equation has the form

$$
u^{\prime \prime}+\left(\frac{c_{0}}{z}+\frac{c_{1}}{z-1}+\frac{c_{2}}{z-\lambda}\right) u^{\prime}+\frac{a b z-q}{z(z-1)(z-\lambda)} u=0,
$$

where $c_{0}, c_{1}, c_{2}, a, b$ and $q$ are complex parameters satisfying the relation

$$
c_{0}+c_{1}+c_{2}=a+b+1 \text {. }
$$

More generally, a linear differential equation of the form $u^{\prime \prime}+P u^{\prime}+Q u$ $=0$ can be transformed locally to the form $y^{\prime \prime}+R y=0$ by putting $u(z)=$ $\exp \left(-\int^{z} P(\zeta) d \zeta / 2\right) y(z)$. In the case when $c_{0}=c_{1}=c_{2}=a=b=1$ and $q=(1-t-c(\lambda)) / 2$, Heun's equation takes the form

$$
u^{\prime \prime}+\left(\frac{1}{z}+\frac{1}{z-1}+\frac{1}{z-\lambda}\right) u^{\prime}+\frac{2 z+t+c(\lambda)-1}{2 z(z-1)(z-\lambda)} u=0
$$

and the above-mentioned transformation

$$
u=\frac{y}{\sqrt{z(z-1)(z-\lambda)}}
$$

reduces Heun's equation to our equation (4.5). It is an interesting fact that the factor $\omega(z):=1 / \sqrt{z(z-1)(z-\lambda)}$ forms a basis of the one-dimensional vector space of Abelian differentials on the torus defined as the algebraic curve $w^{2}=$ 
$z(z-1)(z-\lambda)$. In particular, $(\omega \circ A) \cdot A^{\prime}= \pm \omega$ holds for an automorphism $A$ of $Y=\mathbb{C} \backslash\{0,1, \lambda\}$.

Therefore, for a solution $u$ of (7.1) and $A \in \operatorname{Aut}(Y)$, we can see that $A_{1 / 2}^{*} u:=$ $(u \circ A)\left(A^{\prime}\right)^{1 / 2}$ is a solution of (7.1) again.

We now set

$$
\widetilde{F}_{z_{1}}=\left(\begin{array}{ll}
u_{1}^{\prime} & u_{1} \\
u_{2}^{\prime} & u_{2}
\end{array}\right)
$$

where $u_{1}$ and $u_{2}$ are the fundamental solutions of (7.1) at $z_{1}$, namely, the solutions satisfying the initial conditions $u_{1}\left(z_{1}\right)=0, u_{1}^{\prime}\left(z_{1}\right)=1$ and $u_{2}\left(z_{1}\right)=1, u_{2}^{\prime}\left(z_{1}\right)=0$, respectively. For $[\alpha] \in Y\left[z_{1}, z_{2}\right]$ we denote by $\widetilde{H}_{[\alpha]}$ the germ at $z_{2}$ obtained by analytic continuation of the fundamental matrix $\widetilde{F}_{z_{1}}$ along the path $\alpha$ in $Y$. In the same way as in the case of (4.5), we also define the transition matrix $\widetilde{L}[\alpha]=\widetilde{L}_{t}[\alpha]$ by the relation $\widetilde{H}_{[\alpha]}=\widetilde{L}[\alpha] \widetilde{F}_{z_{2}}$ for $[\alpha] \in Y\left[z_{1}, z_{2}\right]$. Then, the similar relations to (i), (ii) and (iii) in Section 3 hold for these quantities. Noting the elementary identity

$$
\omega\left(\begin{array}{l}
y_{1} \\
y_{2}
\end{array}\right)=\left(\begin{array}{cc}
\omega\left(z_{1}\right) & 0 \\
\omega^{\prime}\left(z_{1}\right) & \omega\left(z_{1}\right)
\end{array}\right)\left(\begin{array}{l}
u_{1} \\
u_{2}
\end{array}\right)=K\left(z_{1}\right)\left(\begin{array}{l}
u_{1} \\
u_{2}
\end{array}\right)
$$

for the fundamental solutions of (4.5) and (7.1) at $z_{1}$, where

$$
K(z)=\omega(z)\left(\begin{array}{cc}
1 & 0 \\
\mu(z) & 1
\end{array}\right), \quad \mu(z)=-\frac{1}{2}\left(\frac{1}{z}+\frac{1}{z-1}+\frac{1}{z-\lambda}\right)
$$

we have the fundamental relation $F_{z_{1}} K=K\left(z_{1}\right) \widetilde{F}_{z_{1}}$ near $z_{1} \in Y$. By analytic continuation, we obtain $H_{[\alpha]} K\left(z_{2}\right)=K\left(z_{1}\right) \widetilde{H}_{[\alpha]}$ for $[\alpha] \in Y\left[z_{1}, z_{2}\right]$, in particular,

$$
L[\alpha]=K\left(z_{1}\right) \widetilde{L}[\alpha] K\left(z_{2}\right)^{-1} .
$$

Let $z_{1}$ be a point in $Y=\mathbb{C} \backslash\{0,1, \lambda\}$. We now take curves $\varepsilon_{1}, \varepsilon_{2}, \varepsilon_{3}$ and $\varepsilon_{4}$ in $Y$ starting from $z_{1}$, rounding $0,1, \lambda$ and $\infty$, respectively, once anticlockwise and ending at $z_{1}$, so that $\pi_{1}\left(Y, z_{1}\right)=\left\langle\left[\varepsilon_{1}\right],\left[\varepsilon_{2}\right],\left[\varepsilon_{3}\right],\left[\varepsilon_{4}\right]\right\rangle$ and $\left[\varepsilon_{1}\right]\left[\varepsilon_{2}\right]\left[\varepsilon_{3}\right]\left[\varepsilon_{4}\right]=1$. Then, the next result immediately follows.

Theorem 7.1. Let $l_{t}$ and $\tilde{l}_{t}$ be monodromy homomorphisms of differential equations (4.5) and (7.1) from $\pi_{1}\left(Y, z_{1}\right)$ into $\mathrm{SL}(2, \mathbb{C})$, respectively. We then have $l_{t}\left[\varepsilon_{j}\right]=-\tilde{l}_{t}\left[\varepsilon_{j}\right]$ for $j=0,1,2,3$. In particular, $l_{t}=\tilde{l}_{t}$ on the canonical image of the Fuchsian group $\Gamma_{0}=\Gamma \cap \Gamma^{\prime}$.

Corollary 7.2. The monodromy group for 4.5 is discrete if and only if the monodromy group for (7.1) is.

Since $L\left[\alpha_{r}\right] M_{z_{0}}\left(A_{r}\right)$ is conjugate with $\widetilde{L}\left[\alpha_{r}\right] \widetilde{M}_{z_{0}}\left(A_{r}\right)$ in $\operatorname{GL}(2, \mathbb{C})$, where $\widetilde{M}_{z_{0}}\left(A_{r}\right)$ $=K\left(A_{r}\left(z_{0}\right)\right)^{-1} M_{z_{0}}\left(A_{r}\right) K\left(z_{0}\right)$ for $r=0, \infty$, we obtain the following statement from Theorem 4.3.

Theorem 7.3. For suitable choices of representatives $\tilde{\theta}_{0}$ of $\theta_{0}$ and $\tilde{\theta}_{\infty}$ of $\theta_{\infty}$ in $\mathrm{SU}(1,1)$,

$$
\tilde{\chi}_{t \varphi_{0}}\left(\tilde{\theta}_{0}\right)=\widetilde{L}_{t}\left[\alpha_{0}\right] \widetilde{M}_{z_{0}}\left(A_{0}\right)
$$

and

$$
\tilde{\chi}_{t \varphi_{0}}\left(\tilde{\theta}_{\infty}\right)=\widetilde{L}_{t}\left[\alpha_{\infty}\right] \widetilde{M}_{z_{0}}\left(A_{\infty}\right)
$$

for any $t \in \mathbb{C}$ up to $\mathrm{SL}(2, \mathbb{C})$-conjugacy, where $\varphi_{0}=p_{2}^{*}\left(\psi_{0}\right)$. 


\section{REFERENCES}

1. L. V. Ahlfors, Complex Analysis, 3rd ed., McGraw Hill, New York, 1979. MR 80c:30001

2. A. F. Beardon, Iteration of Rational Functions, Springer-Verlag, 1991. MR 92j:30026

3. L. Bers, Uniformization, moduli and Kleinian groups, Bull. London Math. Soc. 4 (1972), 257-300. MR 50:595

4. A. Douady and C. J. Earle, Conformally natural extensions of homeomorphisms of the circle, Acta Math. 157 (1986), 23-48. MR 87j:30041

5. D. B. A. Epstein and A. Marden, Convex hulls in hyperbolic space, a theorem of Sullivan, and measured pleated surfaces, Analytical and Geometric Aspects of Hyperbolic Space, London Math. Soc. Lecture Notes Series no. 111, Cambridge University Press, 1987, pp. 113-254. MR 89c:52014

6. F. P. Gardiner, Schiffer's interior variation and quasiconformal mappings, Duke Math. 42 (1975), 371-380. MR 52:3519

7. G. H. Hardy and E. M. Wright, An introduction to the theory of numbers, fifth ed., The Clarendon Press, Oxford University Press, New York, 1979. MR 81i:10002

8. J. A. Hempel, The uniformization of the n-punctured sphere, Bull. London Math. Soc. 20 (1988), 97-115. MR 89c:30109

9. E. Hille, Ordinary Differential Equations in the Complex Domain, Wiley-Interscience, 1976. MR 58:17266

10. Y. Imayoshi and M. Taniguchi, An introduction to Teichmüller spaces, Springer-Tokyo, 1992. MR 94b:32031

11. K. Ito, Exotic projective structures and quasi-Fuchsian space, Duke Math. J. 105 (2000), 185-209. MR 2001j:30038

12. L. Keen, Teichmueller spaces of punctured tori: I, II, Complex Variables Theory Appl. 2 (1983), 199-211, and 213-225. MR 85b:32034

13. L. Keen, H. E. Rauch, and A. T. Vasquez, Moduli of punctured tori and the accessory parameter of Lamé's equation, Trans. Amer. Math. Soc. 255 (1979), 201-230. MR 81j:30074

14. L. Keen and C. Series, Pleating invariants for punctured torus groups, Warwick University preprint, 10/1998.

15. tori, Topology 32 (1993), 719-749. MR 95g:32030

16. S. P. Kerckhoff, Lines of minima in Teichmüller space, Duke Math. J. 65 (1992), 187-213. MR 93b:32027

17. Y. Komori and C. Series, Pleating coordinates for the Earle embedding, Ann. Fac. Sci. Toulouse, VI. Sér., Math. 10 (2001), 69-105. MR 2004b:32021

18. Y. Komori, T. Sugawa, M. Wada, and Y. Yamashita, Drawing Bers embeddings of the Teichmüller space of once-punctured tori, preprint.

19. I. Kra, A generalization of a theorem of Poincaré, Proc. Amer. Math. Soc. 27 (1971), 299-302. MR 46:347

20. - Accessory parameters for punctured spheres, Trans. Amer. Math. Soc. 313 (1989), 589-617. MR 89j:30062

21. I. Kra and B. Maskit, Remarks on projective structures, Riemann Surfaces and Related Topics, Ann. of Math. Studies, no. 97, 1981, pp. 343-359. MR 83f:30042

22. S. L. Krushkal, Teichmüller spaces are not starlike, Ann. Acad. Sci. Fenn. Ser. A I Math. 20 (1995), 167-173. MR 96f:32031

23. I. Laine and T. Sorvali, Local solution of $w^{\prime \prime}+A(z) w=0$ and branched polymorphic functions, Result. Math. 10 (1986), 107-129. MR 87j:30109

24. B. Maskit, On a class of Kleinian groups, Ann. Acad. Sci. Fenn. Ser. A I Math. 442 (1969), 1-8. MR 40:5857

25. C. McMullen, Rational maps and Kleinian groups, Proceedings of the International Congress of Mathematicians (Kyoto, 1990), Springer, 1991, pp. 889-900. MR 93h:57024

26. C_ Complex earthquakes and Teichmüller theory, J. Amer. Math. Soc. 11 (1998), 283320. MR 98i:32030

27. Y. Minsky, The classification of punctured-torus groups, Ann. of Math. (2) 149 (1999), 559626. MR 2000f:30028

28. H. Miyachi, Cusps in complex boundaries of one-dimensional Teichmüller spaces, Conform. Geom. Dyn. 7 (2003), 103-151. 
29. S. Nag, The Complex Analytic Theory of Teichmüller Spaces, Wiley, New York, 1988. MR 89f:32040

30. Y. Okumura, Lifting problem of Fuchsian groups and a characterization of simple dividing loops on Riemann surfaces, in preparation.

31. S. M. Pizer, Numerical Computing and Mathematical Analysis, Science Research Associates, 1975.

32. R. M. Porter, Computation of a boundary point of Teichmüller space, Bol. Soc. Mat. Mexicana 24 (1979), 15-26. MR 81j:32026

33. A. Ronveaux, Heun's Differential Equations, Oxford University Press, 1995. MR 98a:33005

34. H. L. Royden, Automorphisms and isometries of Teichmüller space, Advances in the Theory of Riemann Surfaces, Ann. of Math. Stud. 66, Princeton Univ. Press, 1971, pp. 369-383. MR 44:5452

35. H. Shiga, Projective structures on Riemann surfaces and Kleinian groups, J. Math. Kyoto Univ. 27 (1987), 433-438. MR 88k:30056

36. H. Shiga and H. Tanigawa, Projective structures with discrete holonomy representations, Trans. Amer. Math. Soc. 351 (1999), 813-823. MR 99d:32025

37. T. Sugawa, Estimates of hyperbolic metric with applications to Teichmüller spaces, Kyungpook Math. J. 42 (2002), 51-60. MR 2003g:30084

38. M. Toki, On nonstarlikeness of Teichmüller spaces, Proc. Japan Acad. Ser. A Math. Sci. 69 (1993), 58-60. MR 94h:32039

39. P. Tukia, Quasiconformal extension of quasisymmetric mappings compatible with a Möbius group, Acta Math. 154 (1985), 153-193. MR 86f:30024

40. D. J. Wright, The shape of the boundary of Maskit's embedding of the Teichmüller space of once punctured tori, unpublished manuscript.

Department of Mathematics, Osaka City University, Sugimoto 3-3-138 Sumiyoshi-Ku, OSAKA, 558-8585 JAPAN

E-mail address: komori@sci.osaka-cu.ac.jp

Department of Mathematics, Graduate School of Science, Hiroshima University, 13-1 Kagamiyama, Higashi-Hiroshima, 739-8526 Japan

E-mail address: sugawa@math.sci.hiroshima-u.ac.jp 